2016

\title{
HMOG: New Behavioral Biometric Features for Continuous Authentication of Smartphone Users
}

Zdenka Sitova

New York Intitute Technol, New York, NY 10023 USA;

Jaroslav Sedenka

New York Intitute Technol, New York, NY 10023 USA;

Qing Yang

College of William \& Mary, Williamsburg, VA 23185 USA

Ge Peng

College of William \& Mary, Williamsburg, VA 23185 USA

Gang Zhou

College of William \& Mary, Williamsburg, VA 23185 USA

See next page for additional authors

Follow this and additional works at: https://scholarworks.wm.edu/aspubs

\section{Recommended Citation}

Sitová, Z., Šeděnka, J., Yang, Q., Peng, G., Zhou, G., Gasti, P., \& Balagani, K. S. (2016). HMOG: New behavioral biometric features for continuous authentication of smartphone users. IEEE Transactions on Information Forensics and Security, 11(5), 877-892. 


\section{Authors}

Zdenka Sitova, Jaroslav Sedenka, Qing Yang, Ge Peng, Gang Zhou, and Paolo Gasti 


\title{
HMOG: New Behavioral Biometric Features for Continuous Authentication of Smartphone Users
}

\author{
Zdeňka Sitová, Jaroslav Šeděnka, Qing Yang, Ge Peng, Gang Zhou, Senior Member, IEEE, \\ Paolo Gasti, Member, IEEE, and Kiran S. Balagani, Member, IEEE
}

\begin{abstract}
We introduce hand movement, orientation, and grasp (HMOG), a set of behavioral features to continuously authenticate smartphone users. HMOG features unobtrusively capture subtle micro-movement and orientation dynamics resulting from how a user grasps, holds, and taps on the smartphone. We evaluated authentication and biometric key generation (BKG) performance of HMOG features on data collected from 100 subjects typing on a virtual keyboard. Data were collected under two conditions: 1) sitting and 2) walking. We achieved authentication equal error rates (EERs) as low as $\mathbf{7 . 1 6 \%}$ (walking) and $\mathbf{1 0 . 0 5 \%}$ (sitting) when we combined HMOG, tap, and keystroke features. We performed experiments to investigate why HMOG features perform well during walking. Our results suggest that this is due to the ability of HMOG features to capture distinctive body movements caused by walking, in addition to the handmovement dynamics from taps. With BKG, we achieved the EERs of $15.1 \%$ using HMOG combined with taps. In comparison, BKG using tap, key hold, and swipe features had EERs between $25.7 \%$ and $34.2 \%$. We also analyzed the energy consumption of HMOG feature extraction and computation. Our analysis shows that HMOG features extracted at a $16-\mathrm{Hz}$ sensor sampling rate incurred a minor overhead of $7.9 \%$ without sacrificing authentication accuracy. Two points distinguish our work from current literature: 1) we present the results of a comprehensive evaluation of three types of features (HMOG, keystroke, and tap) and their combinations under the same experimental conditions and 2) we analyze the features from three perspectives (authentication, BKG, and energy consumption on smartphones).
\end{abstract}

Index Terms-Behavioral biometrics, continuous authentication, biometric key generation, energy evaluation, HMOG.

Manuscript received December 20, 2014; revised May 21, 2015; accepted November 3, 2015. Date of publication December 8, 2015; date of current version February 24, 2016. This work was supported in part by a 2013 New York Institute of Technology Institutional Support for Research and Creativity (ISRC) grant, and in part by the Defense Advanced Research Projects Agency Active Authentication under Grant FA8750-13-2-0266. The work of G. Zhou was supported by the National Science Foundation CAREER Grant CNS1253506. The associate editor coordinating the review of this manuscript and approving it for publication was Dr. Hafiz Malik. (Corresponding author: Kiran S. Balagani).

Z. Sitová and J. Šeděnka were with New York Intitute of Technology, New York, NY 10023 USA, on leave from Masaryk University, Brno 601 77, Czech Republic (e-mail: sitovaz@mail.muni.cz; sedenka@mail.muni.cz).

Q. Yang, G. Peng, and G. Zhou are with the College of William and Mary, Williamsburg, VA 23185 USA (e-mail: qyang@cs.wm.edu; gpeng@cs.wm.edu; gzhou@cs.wm.edu).

P. Gasti and K. S. Balagani are with the New York Institute of Technology, New York, NY 10023 USA (e-mail: pgasti@nyit.edu; kbalagan@ nyit.edu).

This paper has supplementary downloadable material available at http://ieeexplore.ieee.org., provided by the author.

Color versions of one or more of the figures in this paper are available online at http://ieeexplore.iee.org.

Digital Object Identifier 10.1109/TIFS.2015.2506542

\section{INTRODUCTION}

$\mathbf{C}$ URRENTLY, popular smartphone authentication mechanisms such as PINs, graphical passwords, and fingerprint scans offer limited security. They are susceptible to guessing [1] (or spoofing [2] in the case of fingerprint scans), and to side channel attacks such as smudge [3], reflection [4], and video capture [5] attacks. Additionally, a fundamental limitation of PINs, passwords, and fingerprint scans is that they are well-suited for one-time authentication, and therefore are commonly used to authenticate users at login. This renders them ineffective when the smartphone is accessed by an adversary after login. Continuous or active authentication addresses these challenges by frequently and unobtrusively authenticating the user via behavioral biometric signals, such as touchscreen interactions [6], hand movements and gait [7], [8], voice [9], and phone location [10].

In this paper, we present Hand Movement, Orientation, and Grasp (HMOG), a new set of behavioral biometric features for continuous authentication of smartphone users. HMOG uses accelerometer, gyroscope, and magnetometer readings to unobtrusively capture subtle hand micro-movements and orientation patterns generated when a user taps on the screen.

HMOG features are founded upon two core building blocks of human prehension [11]: stability grasp, which provides stability to the object being held; and precision grasp, which involves precision-demanding tasks such as tapping a target. We view the act of holding a phone as a stability grasp and the act of touching targets on the touchscreen as a precision grasp. We hypothesize that the way in which a user "distributes" or "shares" stability and precision grasps while interacting with the smartphone results in distinctive movement and orientation behavior. The rationale for our hypothesis comes from the following two bodies of research.

First, there is evidence (see [12]-[14]) that users have postural preferences for interacting with hand-held devices such as smartphones. Depending upon the postural preference, it is possible that the user can have her own way of achieving stability and precision-for example, the user can achieve both stability and precision with one hand if the postural preference involves holding and tapping the phone with the same hand; or distribute stability and precision between both hands, if the posture involves using both hands for holding and tapping; or achieve stability with one hand and precision with the other.

Second, studies in ergonomics, biokinetics, and humancomputer interaction have reported that handgrip strength 
strongly correlates with an individual's physiological and somatic traits like hand length, handedness, age, gender, height, body mass, and musculature (see, e.g., [15]-[17]). If the micro-movements caused by tapping reflect an individual's handgrip strength, then the distinctiveness of HMOG may have roots, at least in part, in an individual's distinctive physiological and somatic traits.

Motivated by the above, we designed 96 HMOG features and evaluated their continuous user authentication and biometric key generation performance during typing. Because walking has been shown to affect typing performance [18], we evaluated HMOG under both walking and sitting conditions.

\section{A. Contributions and Novelty of This Work}

1) New HMOG Features for Continuous Authentication: We propose two types of HMOG features: resistance features, which measure the micro-movements of the phone in response to the forces exerted by a tap gesture; and stability features, which measure how quickly the perturbations in movement and orientation, caused by tap forces, dissipate. Our extensive evaluation of HMOG features on a dataset of 100 users $^{1}$ who typed on the smartphone led to the following findings: (1) HMOG features extracted from accelerometer and gyroscope signals outperformed HMOG features from magnetometer; (2) Augmenting HMOG features with tap characteristics (e.g., tap duration and contact size) lowered equal error rates (EERs): from $14.34 \%$ to $11.41 \%$ for sitting, and from $14.73 \%$ to $8.53 \%$ for walking. This shows that combining tap information with HMOG features considerably improves authentication performance; and (3) HMOG features complement tap and keystroke dynamics features, especially for low authentication latencies at which tap and keystroke dynamics features fare poorly. For example, for 20-second authentication latency, adding HMOG to tap and keystroke dynamics features reduced the equal error rate from $17.93 \%$ to $11.74 \%$ for walking and from $19.11 \%$ to $15.25 \%$ for sitting.

2) Empirical Investigation Into Why HMOG Authentication Performs Well During Walking: HMOG features achieved lower authentication errors (13.62\% EER) for walking compared to sitting (19.67\% EER). We investigated why HMOG had a superior performance during walking by comparing the performance of HMOG features during taps and between taps (i.e., the segments of the sensor signal that lie between taps). Our results suggest that the higher authentication performance during walking can be attributed to the ability of HMOG features to capture distinctive movements caused by walking in addition to micro-movements caused by taps.

3) BKG With HMOG Features: BKG is closely related to authentication, but has a different objective: to provide cryptographic access control to sensitive data on the smartphone. We believe that designing a secure BKG scheme on smartphones is very important, because the adversary is usually assumed to have physical access to the device, and therefore cryptographic keys must not be stored on the smartphone's

\footnotetext{
${ }^{1}$ We made the dataset available at http://www.cs.wm.edu/ qyang/hmog.html. We also described the data and its release in [19].
}

memory-but rather generated from biometric signals and/or passwords.

To our knowledge, we are the first to evaluate $\mathrm{BKG}$ on smartphones. We instantiated BKG using normalized generalized Reed-Solomon codes in Lee metric. (See section VI for formulation and evaluation.) We compared BKG on HMOG to BKG on tap, key hold, and swipe features under two metrics: equal error rate (EER) and guessing distance.

Our results on BKG can be summarized as follows: we achieved lower EERs with HMOG features compared to key hold, tap, and swipe features in both walking and sitting conditions. For walking, EER of HMOG-based BKG was $17 \%$, vs. $29 \%$ with key hold and $28 \%$ with tap features. By combining HMOG and tap features, we achieved $15.1 \%$ EER. For sitting, we obtained an EER of $23 \%$ with HMOG features, $26 \%$ with tap features, and $20.1 \%$ by combining both. In contrast, we obtained 34\% EER with swipes. HMOG features also provided higher guessing distance (i.e., 2.9 for walking, and 2.8 for sitting) than all other features extracted from our dataset (1.9 for taps and for key holds in walking and 1.6 for taps in sitting conditions).

4) Energy Consumption Analysis of HMOG Features: Because smartphones are energy constrained, it is crucial that a continuous user authentication method consumes as little energy as possible, while maintaining the desired level of authentication performance. To evaluate the feasibility of HMOG features for continuous authentication on smartphones, we measured the energy consumption of accelerometer and gyroscope, sampled at $100 \mathrm{~Hz}, 50 \mathrm{~Hz}, 16 \mathrm{~Hz}$ and $5 \mathrm{~Hz}$. We then measured the energy required for HMOG feature computation from sensor signals, and reported the tradeoff between energy consumption and EER.

Our analysis shows that a balance between authentication performance and energy overhead can be achieved by sampling HMOG features at $16 \mathrm{~Hz}$. The energy overhead with $16 \mathrm{~Hz}$ is $7.9 \%$, compared to $20.5 \%$ with $100 \mathrm{~Hz}$ sampling rate, but comes with minor increase (ranging from $0.4 \%$ to $1.8 \%$ ) in EERs. However, by further reducing the sampling rate to $5 \mathrm{~Hz}$, we observed a significant increase in EER (11.0\% to $14.1 \%)$.

\section{B. Organization}

We present the description of HMOG features in Section II, and details on our dataset in Section III. In sections IV and V, we describe the authentication experiments and present results. We introduce and evaluate BKG on HMOG in Section VI. We analyze the energy consumption of HMOG features in Section VII. In Section VIII, we review related research. We conclude in Section IX.

\section{DESCRIPTION OF HMOG FEATURES}

We define two types of HMOG features: grasp resistance and grasp stability. These features are computed from data collected using three sensors: accelerometer, gyroscope, and magnetometer. Because HMOG features aim to capture the subtle micro-movements and orientation patterns of a user while tapping on the screen, we extract HMOG features from signals collected during or close to tap events. Computation of grasp stability and resistance features is discussed next. 
TABLE I

NOTATION

\begin{tabular}{|c|l|}
\hline Sensor & Accelerometer or Gyroscope or Magnetometer. \\
\hline$X, Y, Z$ & Time series of sensor readings in $x, y$, and $z$ axes respectively \\
\hline$Z_{1}, \ldots, Z_{n}$ & $\begin{array}{l}\text { Individual sensor readings in } z \text { axis collected at time } \\
t_{1}, \ldots, t_{n} \text { respectively }\end{array}$ \\
\hline$M$ & $\begin{array}{l}\text { Time series of magnitude of sensor reading, where each } \\
\text { element } M_{i} \text { is computed as } \sqrt{\left(X_{i}^{2}+Y_{i}^{2}+Z_{i}^{2}\right)}\end{array}$ \\
\hline$t_{\text {start }}$ & Start time of a tap event \\
\hline$t_{\text {end }}$ & End time of a tap event \\
\hline$t_{\text {max_in_tap }}$ & $\begin{array}{l}\text { Time between } t_{\text {start }} \text { and } t_{\text {end }} \text { at which the reading from a } \\
\text { sensor reaches its highest value }\end{array}$ \\
\hline$t_{\text {min }}$ & Time when stability is achieved after the tap event has ended \\
\hline$t_{\text {before } \text { fenter }_{\text {cen }}}$ & Center of the 100 ms window before a tap \\
\hline$t_{\text {after_center }}$ & Center of the $100 \mathrm{~ms}$ window after a tap \\
\hline avg100msBefore & $\begin{array}{l}\text { Average of sensor readings in a } 100 \mathrm{~ms} \text { window before start } \\
\text { time and after end time, respectively }\end{array}$ \\
\hline avg 10 msAfter & Average of readings during tap events \\
\hline$t_{\text {min }}$ & Time when stability is achieved after the tap event has ended \\
\hline
\end{tabular}

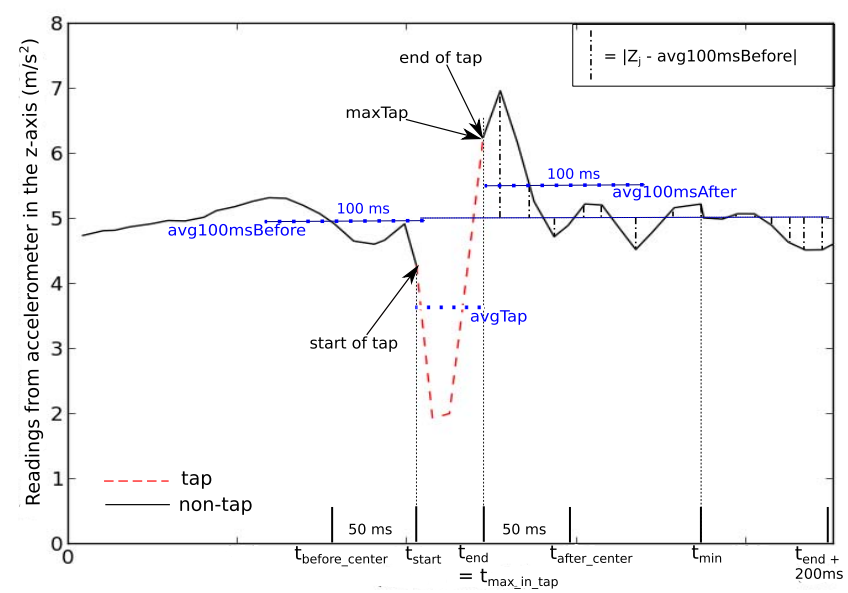

Fig. 1. Illustration of key variables for computing grasp resistance features 3-5 and grasp stability features 1-3.

\section{A. Grasp Resistance Features}

Grasp resistance features measure the resistance of a hand grasp to the forces (or pressures) exerted by touch/gesture events. We quantify resistance as the change, or perturbation, in movement (using readings from accelerometer), orientation (from gyroscope) and magnetic field (from magnetometer), caused by a tap event.

We extracted five grasp resistance features from accelerometer, gyroscope, and magnetometer, over four dimensions (magnitude, $x, y$, and $z$ axes), leading to $5 \times 3 \times 4=60$ features. For simplicity of exposition, we describe grasp resistance features only on the $z$ axis. We also extracted the same features from $X, Y$, and $M$. Our notation is summarized in Table I. Figure 1 illustrates variables used in features 3 through 5 .

1) Mean of $Z$ during taps.

2) Standard deviation of $Z$ during taps.

3) Difference in $Z$ readings before and after a tap event. Let avg100msBefore be the average of $Z$ readings in a $100 \mathrm{~ms}$ window before tap start time, and avg100msAfter be the average of $Z$ readings in a $100 \mathrm{~ms}$ window after tap end time. We calculated this

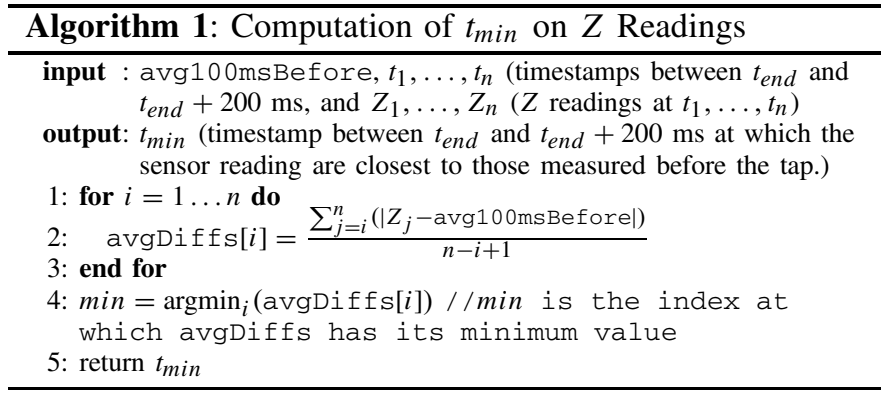

feature as the difference between avg100msAfter and avg100msBefore.

4) Net change in $Z$ readings caused by a tap. Let avgTap be the average of $Z$ readings during a tap event. We calculate this feature as avgTap - avg100msBefore.

5) Maximum change in $Z$ readings caused by a tap. Let maxTap be the maximum $Z$ reading during a tap event. This feature is calculated as maxTap avg10 0msBefore.

\section{B. Grasp Stability Features}

Stability features quantify how quickly the perturbations caused by a finger-force from a tap event disappear after the tap event is complete. We compute grasp stability features as follows: (Figure 1 illustrates variables used in the features.)

1) Time duration to achieve movement and orientation stability after a tap event. Let $t_{\text {end }}$ denote the end time of the tap event, and $t_{\min }$ the time when stability is achieved after the tap event has ended, computed as shown in Algorithm 1. This feature is calculated as $t_{\min }-t_{\text {end }}$.

2) Normalized time duration for mean sensor value to change from before tap to after tap event, calculated as:

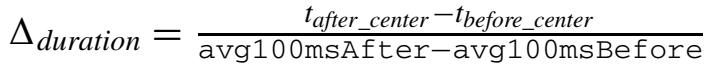

where $t_{\text {after_center }}$ is the center of the $100 \mathrm{~ms}$ window after a tap event, and thefore_center is the center of the $100 \mathrm{~ms}$ window before the tap event.

3) Normalized time duration for mean sensor values to change from maxTap to avg100msAfter in response to a tap event, calculated as:

$$
\Delta_{\text {max_to_avg }}=\frac{t_{\text {after_center }}-t_{\text {max_in_tap }}}{\operatorname{avg100msAfter-maxTap}}
$$

where maxTap is the maximum sensor value during a tap, and $t_{\text {max_in_tap }}$ is the time when this value occurred. We extracted the above three grasp stability features for three sensors and four types of sensor readings $(X, Y, Z$ and $M)$, for a total of $3 \times 3 \times 4=36$ features.

Complexity of computing HMOG features is linear $(\mathrm{O}(n))$ in the sampling frequency, except for Grasp Stability Feature 1, which is quadratic $\left(\mathrm{O}\left(n^{2}\right)\right)$.

\section{DATASET}

To evaluate HMOG features, we used sensor data collected from 100 smartphone users (53 male, 47 female) during eight 


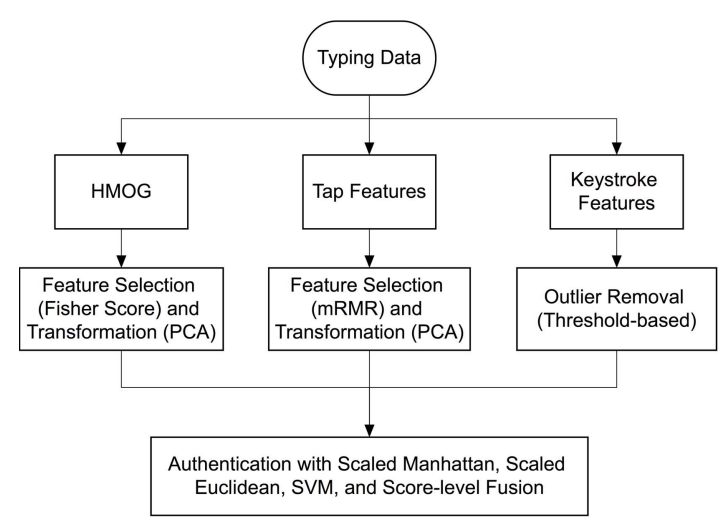

Fig. 2. Flow-diagram depicting our experiment workflow.

free text typing sessions [19]. ${ }^{2}$ Users answered three questions per session, typing at least 250 characters for each answer. In four sessions, the users typed while sitting. In another four sessions, users typed while walking in a controlled environment.

For each user, we collected an average of 1193 taps per session (standard deviation: 303) and 1019 key presses (standard deviation: 258). The average duration of a session was 11.6 minutes, with a standard deviation of 4.6 minutes. Data was collected using the same smartphone model (Samsung Galaxy S4). We used a total of ten Samsung Galaxy S4. Data was collected over multiple days, and the same user might have received a different device during each visit.

We recorded accelerometer, gyroscope and magnetometer sensor readings (sampling rate $100 \mathrm{~Hz}$ ) as well as raw touch data collected from the touchscreen, touch gestures (e.g., tap, scale, scroll, and fling), key press, and key release latencies on the virtual keyboard. Due to security concerns, Android OS forbids third-party applications to access touch and key press data generated on the virtual keyboard. Therefore, we designed a virtual keyboard for data collection that mimicked the look, feel, and functionality of default Android keyboard, including the autocorrect and autocomplete options, which the users were free to use.

During data collection users were allowed to choose the orientation of the smartphone (i.e., landscape or portrait). Because less than 20 users typed in landscape orientation, we performed all authentication experiments with data collected in portrait mode.

\section{Evaluation of HMOG Features}

Our experiment workflow involves: (1) computing features from data collected during typing; (2) performing feature selection; (3) performing feature transformation (PCA); (4) performing outlier removal; and (5) performing authentication using Scaled Manhattan, Scaled Euclidean, SVM verifiers, and score-level fusion. Figure 2 summarizes the experiment workflow.

\footnotetext{
${ }^{2}$ Our dataset is available at http://www.cs.wm.edu/ qyang/hmog.html
}

\section{A. Design of Authentication Experiments}

1) 1-Class Verifiers: We performed verification experiments using three verifiers [20]: scaled Manhattan (SM), scaled Euclidian (SE), and 1-class SVM. (Henceforth, we use "SVM" to refer to "1-class SVM".) We chose these verifiers because previous work on behavioral authentication has shown that they perform well. For instance, SM and SVM were top performers in a study on keystroke authentication of desktop users by Killourhy and Maxion [20]. SVM performed well in experiments on touch-based authentication of smartphone users by Serwadda et al. [21]. SE is a popular verifier in biometrics (see for example [22], [23]).

Parameter tuning was not required for SM and SE. However, for SVM [24], we used RBF kernel and performed a grid search to find the parameters (for $\gamma$, we searched through $2^{-13}, 2^{-11}, 2^{-9}, \ldots, 2^{13}$; and for $v$, we searched through 0.01 , $0.03,0.05,0.1,0.15$ and 0.5$)$. We used cross-validation to choose the parameter values (see Section IV-C).

We did not include 2-class verifiers in our evaluation. To train a 2-class verifier, in addition to data from smartphone owner, biometric data from other users (non-owners) is required. Because sharing of biometric information between smartphone users leads to privacy concerns, we believe that 1-class verifiers are more suitable for smartphone authentication. (A similar argument was made in [25].)

2) Training and Testing: For experiments in sitting and walking conditions, we used the first two sessions for training and the remaining two for testing. We extracted HMOG features during each tap. Thus, each training/testing vector corresponded to one tap. With keystroke dynamics features, each training/testing vector corresponded to one key press on the virtual keyboard.

For SM and SE, the template consisted of the feature-wise average of all training vectors. We used user-wise standard deviations for each feature for scaling. We used all training vectors to construct the template (hypersphere) with SVM. Users with less than 80 training vectors were discarded from authentication. As a consequence, ten users failed to enroll (and were not included in our experiments).

We created authentication vectors by averaging test vectors sampled during $t$-seconds scan. We report results for authentication scans of $t=20,40,60,80,100,120$ and 140 seconds. We chose these scan lengths to cover both low and higher authentication latencies. Our preliminary experiments showed that for scans longer than 140 seconds, there is minimal improvement in authentication performance.

3) Quantifying Authentication Performance: We generated two types of scores, genuine (authentication vector was matched against template of the same user) and zero-effort impostor (authentication vector of one user was matched against the template of another). We used population equal error rate (EER) to measure the authentication performance.

\section{B. Comparing HMOG to Other Feature Sets}

We compared the authentication performance of HMOG features with touchscreen tap and keystroke dynamic features (key hold and digraph latencies). 
TABLE II

PARAMETERs EVALUATEd Using Cross-VALIDATION

\begin{tabular}{ll}
\hline Method & Parameter \\
\hline Fisher score & Percentage of the sum of all Fisher scores \\
mRMR & Threshold on the mRMR score \\
PCA & Percentage of total variance \\
SVM & $\gamma, \nu$ \\
\hline
\end{tabular}

1) Touchscreen Features From Tap Events: We extracted 11 commonly used touchscreen-based features for tap events (see Table VIII). Some papers (e.g., [6], [21]) defined these features for swipes, while we extracted them from taps due to very low availability of swipes during typing, and to provide a more meaningful comparison with HMOG features, which are collected during taps. The features we extracted are:

- Duration of the tap

- Contact size features: mean, median, standard deviation, 1st, 2nd and 3rd quartile, first contact size of a tap, minimum and maximum of the contact size during the tap (9 features)

- Velocity (in pixels per second) between two consecutive press events belonging to two consecutive taps.

2) Key Hold Features: Key hold latency is the down-up time between press and release of a key. We used 89 key hold features, each corresponding to a key on the virtual keyboard.

3) Digraph Features: Digraph latency is the down-down time between two consecutive key presses. We used digraph features for combinations of the 35 most common keys in our dataset. $^{3}$ Thus we have $35^{2}=1225$ digraph features.

4) Score-Level Fusion: To determine whether HMOG features complement existing feature sets, we combined tap, key hold, digraph and HMOG features using weighted sum score-level fusion. We chose this method because it is simple to implement, and has been shown to perform well in biometrics [26]. We used the technique of Locklear et al. [27] to ensure that weights sum to one and proportion of weights is preserved when scores from some feature sets were missing (e.g. due to lack of accelerometer data). We used grid-search to find the weights which led to the best authentication performance.

\section{Feature Selection, Preprocessing, and Transformation}

To improve authentication performance, we performed feature selection, feature transformation with Principal Component Analysis (PCA), and outlier removal.

1) Parameter Selection: We used 10-fold cross-validation (10-CV) on training data to choose feature selection method (mRMR or Fisher score ranking), as well as to set the parameters for feature selection, PCA, and SVM. The parameters are presented in Table II. We evaluated all parameters independently for each combination of feature set, verifier, authentication scan-length and body-motion condition.

\footnotetext{
${ }^{3}$ All 26 alphabetic keys, 5 keyboard switches (shift, switch between numerical and alphabetical keyboard, delete, done, return) and 4 special characters (space, dot, comma and apostrophe). The availability of other keys in our training data was extremely low ( $<1$ on average per user).
}

For each set of parameter values, 10-CV yielded ten EERs, which we averaged to get an estimate of the EER corresponding to that set of parameter values. We then selected parameter values which had the lowest (average) EER. For $10-\mathrm{CV}$ experiments involving 20 - to 140 -second scan lengths, the sets of parameter values that led to the lowest EERs were not always identical. In this case, we took a majority vote to select the most common parameter values.

2) Feature Selection: During training, we evaluated two feature selection methods: Fisher score ranking [28], and minimum-Redundancy Maximum-Relevance (mRMR) [29]. Our preliminary experiments showed that Fisher score performed better for HMOG features, while mRMR performed well with tap features. With key hold and digraph features, the best performing feature set contained all the features.

Fisher score ranking was computed independently for each HMOG feature as the ratio of between-user to within-user variance. (Higher Fisher score suggests higher discriminability of the corresponding feature.) Using $10-\mathrm{CV}$, we tested feature subsets whose sum of Fisher scores accounted for $80 \%$ to $100 \%$ of the sum of Fisher scores of all features.

We selected HMOG features for each verifier separately. The following parameters for Fisher score ranking provided the best authentication results: $82 \%$ (17 features) for SM during sitting; 81\% (13 features) for SM and SVM during walking; and $80 \%$ (16 features) for SVM during sitting. For SE, we achieved lowest EER by including resistance features only, compared to the feature subset obtained from feature selection. Figure 3 reports the ranking of the features during sitting (3(a)) and walking (3(b)).

For tap features, with SM verifier we achieved the best results with 3 features chosen by mRMR (threshold 0) and for SE and SVM with 2 features (threshold 0.1). The best three features according to mRMR are (in this order): duration of the tap; mean of contact size; and velocity between two consecutive down events.

3) Outlier Removal: For HMOG and tap templates, we evaluated the interquartile outlier removal (i.e., different subsets of the values from the first and fourth quartile are removed). Experiments with SM verifier showed that outlier removal does not improve authentication accuracy, so we did not consider it further in our experiments.

For key hold and digraph latencies, using only outlier removal and not performing feature selection or transformation led to the best results. Outlier removal was done using two parameters: (1) latencies longer than $l \mathrm{~ms}$ were discarded and (2) if a feature occurs less than $m$ times in a user's template, the feature was discarded). The values evaluated for $l$ were 100, 200, 300, 400, 500 and 1000 for key hold and 200, 350, 500,650 and 800 for digraph. For $m$, we experimented with $2,5,10,15,20,40$ and 60 . The best $l$ value was 200 for key hold, and between 350-500 for digraph; the best $m$ value was between 2-60 for key hold and between 2-5 for digraph.

4) Feature Transformation: We used PCA to transform original features into principal components, that were subsequently used in authentication experiments. Our motivation for using PCA are: (1) to remove correlation between features to meet the assumptions in SE and SM, and (2) to reduce 


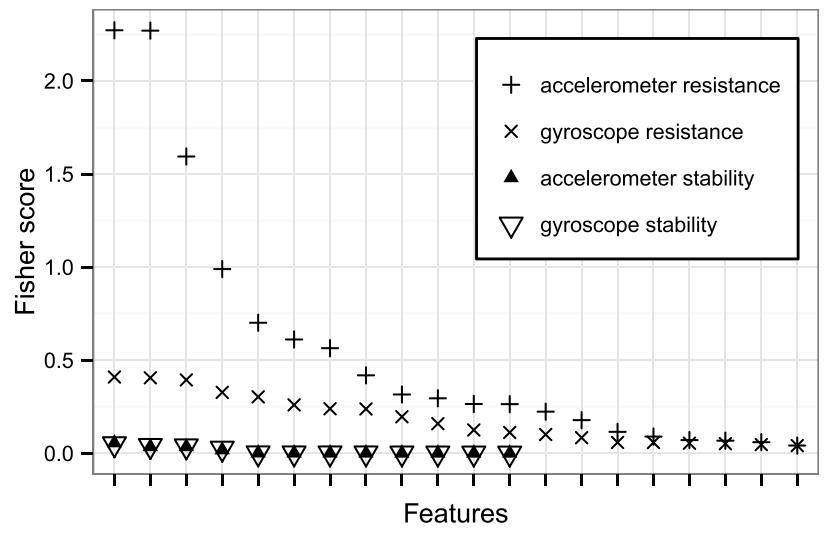

(a)

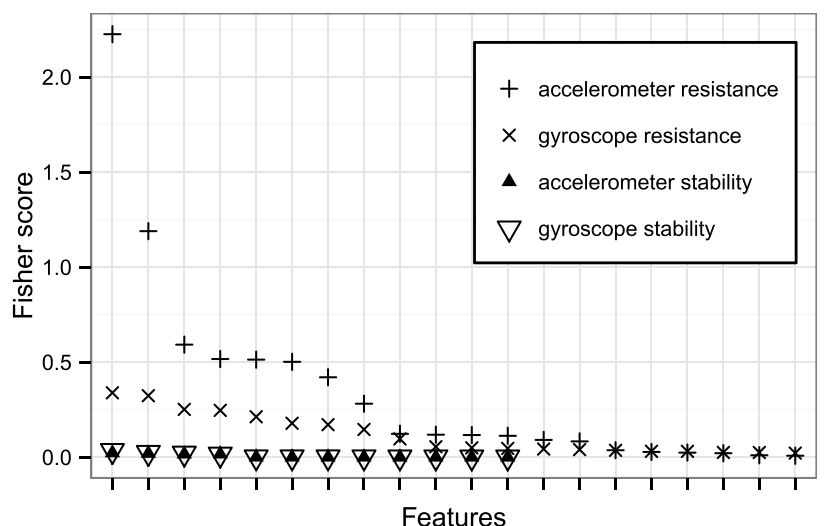

(b)

Fig. 3. HMOG features extracted from accelerometer and gyroscope, sorted by Fisher score computed from training data. Higher scores correspond to features with higher discriminative power. Magnetometer features (not shown) ranked below accelerometer and gyroscope features. (a) Fisher scores of HMOG features during sitting. (b) Fisher scores of HMOG features during walking.

dimensionality by using only those principal components, which explain most of the variance. We performed PCA under two settings: (1) on all features (except magnetometer features, which performed poorly), and (2) on a subset of features selected using Fisher score and mRMR. We performed 10-CV experiments with components explaining $90 \%, 95 \%, 98 \%$, and $100 \%$ of total variance, to set the threshold for dimensionality reduction. PCA improved EER for HMOG features with SE when performed on resistance features, and for SVM during sitting when performed on features selected using Fisher score. PCA performed on all tap features improved results with SM and SE.

\section{Authentication Results}

In this section, we report authentication performance of HMOG features. We compare the performance of HMOG with keystroke and tap features and report results with fusion. ${ }^{4}$ Finally, we present our findings on why HMOG features achieve lower EERs during walking.

\footnotetext{
${ }^{4}$ See Supplement for the number of genuine and impostor scores used for calculating EERs in this paper.
}

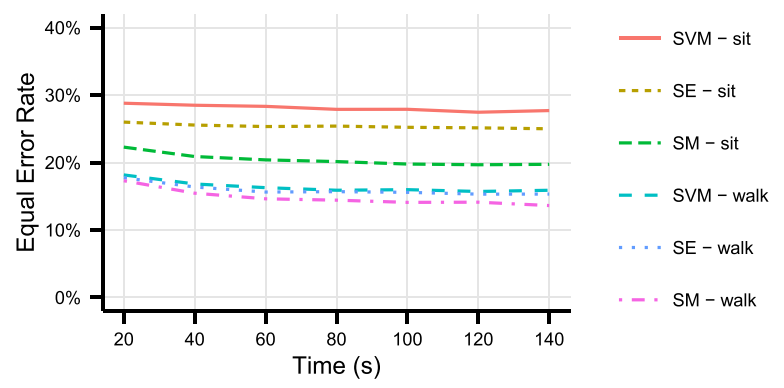

Fig. 4. Comparison of HMOG features in sitting and walking conditions for three verifiers. The reported EERs are with PCA for SE, and for SVM-sitting; and without PCA for SM and SVM-walking. $X$-axis shows authentication time in seconds.

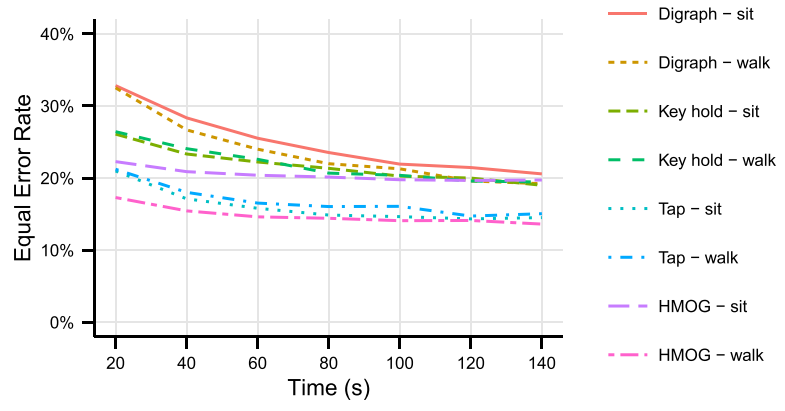

Fig. 5. Comparison of EERs of HMOG with keystroke dynamics (i.e., key hold and digraph) and tap features with SM verifier. $X$-axis shows authentication time in seconds.

\section{A. Performance of HMOG Features}

HMOG features extracted from both accelerometer and gyroscope outperformed those extracted from individual sensors. HMOG features from magnetometer performed consistently worse than accelerometer and gyroscope features with all verifiers, in both sitting and walking conditions. Combining magnetometer features with features from accelerometer and gyroscope did not improve performance.

Resistance features outperformed stability features in both walking and sitting conditions (and also had higher Fisher score, see Figure 3). This suggests that the ability of resistance features to discriminate between users is higher than that of stability features. In fact, feature selection on HMOG with 10-CV resulted in selecting resistance features only. In some cases, using PCA after feature selection further lowered EERs. Table III summarizes the sensors and feature selection/transformation that led to the lowest EERs.

In Figure 4, we show the EERs of all verifiers under sitting and walking conditions, when the authentication scans varied between 20 and 140 seconds. Among the three verifiers, SM overall had lower EERs for both sitting and walking conditions and therefore we present the results only with SM hereafter.

1) Comparison of HMOG With Keystroke Dynamics and Tap Features: Tap features and HMOG features in walking condition performed better than keystroke dynamics features; HMOG in sitting outperforms keystroke dynamics for shorter scans and is comparable for longer scans (see Figure 5).

HMOG features outperformed tap features in walking condition, while tap outperformed HMOG in sitting. 
TABLE III

Summary of LOWEST EERs ACHIEVED USING ONLY HMOG FEATURES

\begin{tabular}{|c|c|c|c|c|}
\hline Verifier & Best Performing Features & Sensors & Sitting & Walking \\
\hline Scaled Manhattan & With Fisher Score Ranking & Accelerometer + Gyroscope & $19.67 \%$ & $13.62 \%$ \\
\hline Scaled Euclidean & With PCA, no Feature Selection & Accelerometer + Gyroscope & $25 \%$ & $15.31 \%$ \\
\hline 1-Class SVM & With Fisher Score Ranking; with PCA for sitting, without PCA for walking & Accelerometer + Gyroscope & $27.45 \%$ & $15.71 \%$ \\
\hline
\end{tabular}

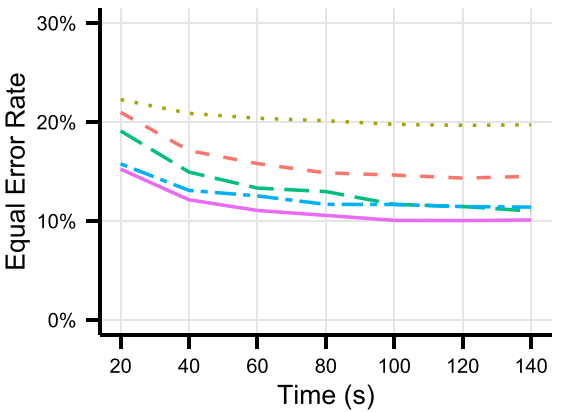

-- Tap

-... HMOG

- - Tap + Key hold +

Digraph

- - $\cdot$ HMOG + Tap

HMOG + Tap + Key hold + Digraph

(a)

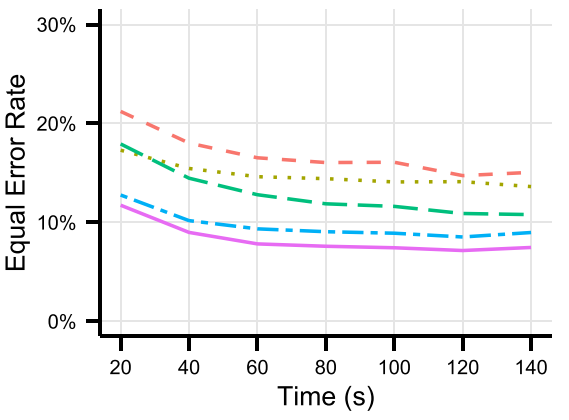

-- Tap

-... HMOG

- - Tap + Key hold +

- - Digraph

$--\cdot$ HMOG + Tap

HMOG + Tap + Key hold + Digraph

(b)

Fig. 6. Score-level fusion of combinations of feature types with SM verifier. $X$ axes show authentication time in seconds. (a) Sitting. (b) Walking.

TABLE IV

SuMMARY OF LOWEST EERS ACHIEVED WITH SCORE-LEVEL FusION OF HMOG,TAP, AND KEYSTROKE DYNAMICS (KD) FEATURES

\begin{tabular}{|c|c|c|}
\hline Score-Level Fusion with SM Verifier & Sitting & Walking \\
\hline HMOG, Tap, and Keystroke Dynamics & $\mathbf{1 0 . 0 5} \%$ & $\mathbf{7 . 1 6} \%$ \\
\hline HMOG and Tap & $11.41 \%$ & $8.53 \%$ \\
\hline Tap and KD & $11.02 \%$ & $10.79 \%$ \\
\hline
\end{tabular}

The performance of tap and keystroke dynamics features did not change significantly between sitting and walking. However, the performance of HMOG improved considerably (up to $6.11 \%$ ) during walking.

2) Fusion of HMOG, Tap, and Keystroke Features: We used SM verifier and performed score-level fusion with the following feature combinations: $\{\mathrm{HMOG}$, tap, keystroke dynamics\}; \{tap, keystroke dynamics\}; and \{tap, HMOG\}. Detailed fusion results for sitting and walking conditions are presented in figures 6(a) and 6(b), respectively. The lowest EERs achieved with fusion are summarized in Table IV,v and the corresponding DET curves for fusion on 60- and 120-second scan lengths are shown in Figure 7.

Our results show that: (1) for both walking and sitting conditions, score-level fusion of all signals led to the lowest EER; and (2) fusing HMOG with tap features led to a decrease in EERs and either outperformed (in the case of walking and shorter scans in sitting) or was comparable (in the case of longer scans in sitting) to fusion of tap and keystroke dynamics (see figures 6(a)) and 6(b)). Both (1) and (2) indicate that HMOG provides additional distinctiveness to that of tap and keystroke dynamics, especially in walking condition.

\section{B. Why HMOG Features Perform Better During Walking}

We investigated why HMOG features performed better during walking. Specifically, we investigated whether the high authentication accuracies of HMOG features during walking were due to hand movements caused by taps, or due to movements caused by walking, or a combination of both.

1) Experiment Setup: We extracted 64 HMOG features from two segments of an accelerometer/gyroscope signal: (1) during tap, as discussed in previous sections; and (2) between taps, in which HMOG features were extracted when the user was not tapping the screen (see Figure 8). In (2), the signal between taps was segmented into non-overlapping blocks of $91 \mathrm{~ms}$; one HMOG feature vector was extracted from each block. We selected $91 \mathrm{~ms}$ as the block size because it was the median duration of a tap in our training data. This ensured that the number of sensor readings used to extract a HMOG feature vector between and during tap remained same.

HMOG features extracted during taps use sensor readings from $100 \mathrm{~ms}$ before and $200 \mathrm{~ms}$ after a tap event (see Section II). We extracted HMOG features between taps starting $300 \mathrm{~ms}$ after a tap until $300 \mathrm{~ms}$ before the next tap, to avoid any overlap between during and between HMOG features.

The average number of the training vectors per user for HMOG during taps was 1122 for sitting, and 1186 for walking. For between taps, it was 7692 for sitting and 7462 for walking. The average number of testing vectors per user for HMOG features during taps was 897 for sitting and 972 for walking. For between taps, it was 5885 for sitting and 5768 for walking. Verification experiments were performed using SM.

2) Performance of HMOG Features Extracted During vs. Between Taps: We compared HMOG features extracted during taps with the same features extracted between taps for sitting and walking conditions. For sitting, HMOG features extracted during taps performed consistently better than those extracted between taps (see EERs in Figure 9). This indicates that HMOG features were able to capture distinctive hand micromovement patterns when the users tapped on the phone. Similarly, for walking, HMOG features extracted during taps performed better than those extracted between taps (see EERs in Figure 9). This again indicates that HMOG features capture 


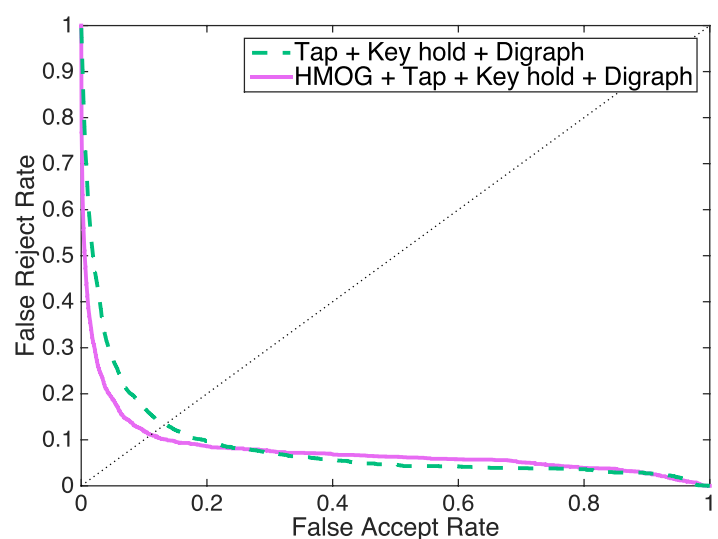

(a)

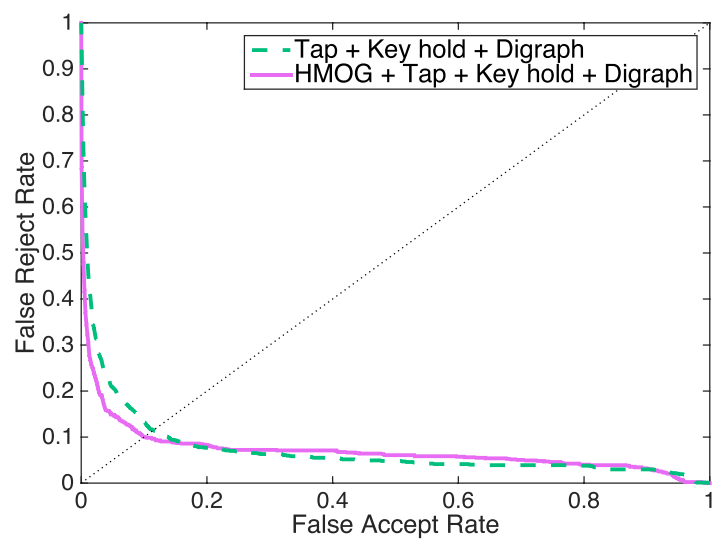

(c)

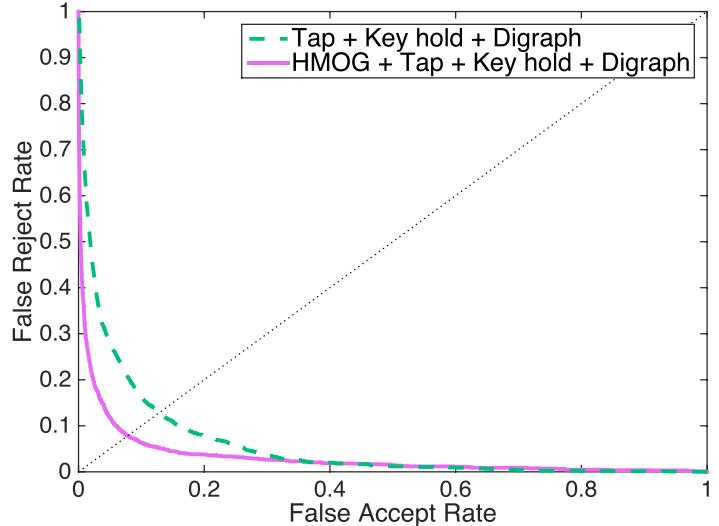

(b)

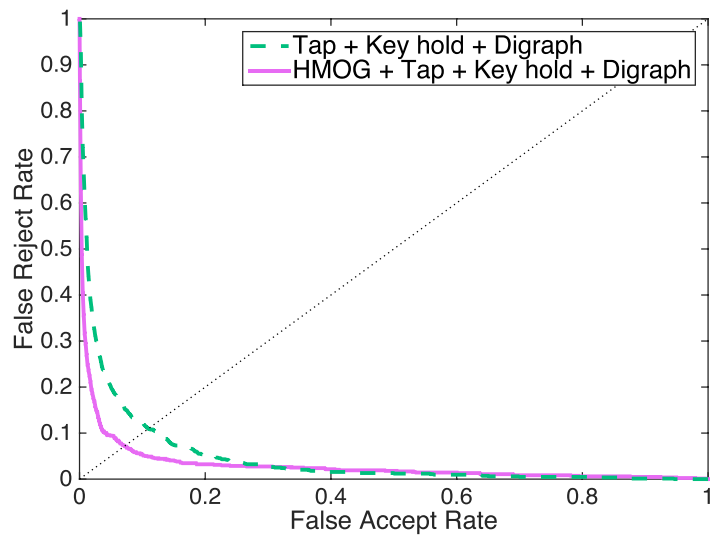

(d)

Fig. 7. DET curves for fusion of all feature types including and excluding HMOG. The scan lengths are 60- and 120-seconds. (a) Sitting (60-second scans). (b) Walking (60-second scans). (c) Sitting (120-second scans). (d) Walking (120-second scans).

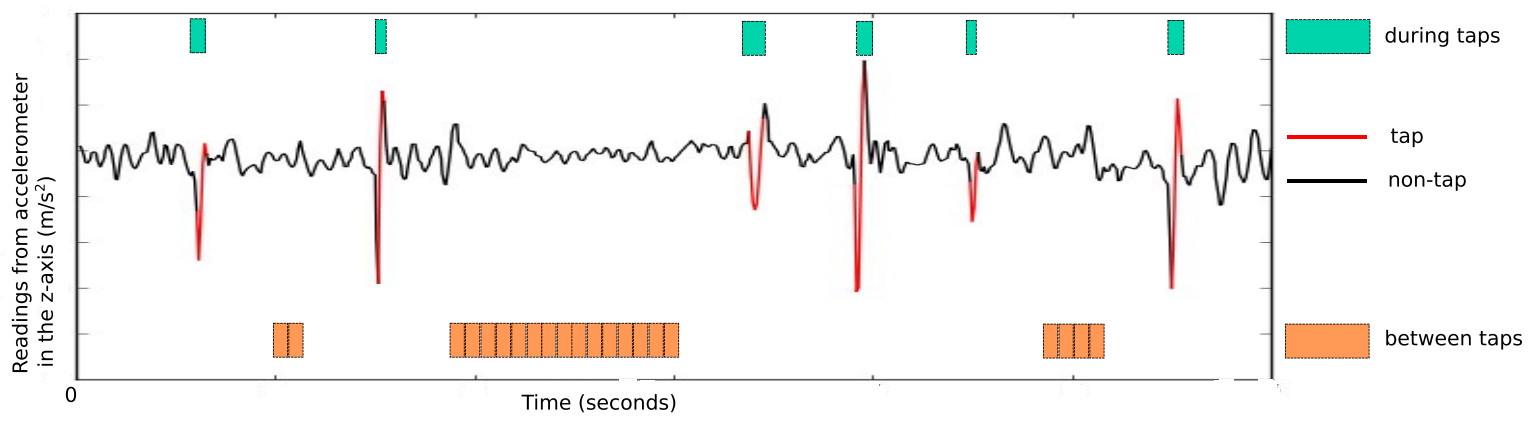

Fig. 8. HMOG features extracted during and between taps. The figure shows a sample of readings from the z-axis of accelerometer in sitting condition.

user's distinctive hand micro-movement patterns when the user is tapping, regardless of the motion condition.

3) Impact of Walking on HMOG Features Extracted Between Taps: HMOG features extracted between taps during walking outperformed the same when extracted during sitting (see between tap EERs for sitting and walking in Figure 9). This indicates that HMOG features capture distinctive movements induced by walking, even in the absence of tap activity.

Supported by the above results, the high authentication accuracies achieved by HMOG features during walking can be jointly attributed to: (a) the distinctiveness in hand movements caused by tap activity and (b) the distinctiveness in movements caused by walking.

\section{Biometric Key Generation FROM HMOG FEATURES}

In this section, we evaluate the performance of HMOG features for biometric key generation (BKG). For this purpose, we introduce our BKG construction, which extends and generalizes the fuzzy commitment scheme of Juels and Wattenberg [30]. While the technique in [30] operates on features represented using a single bit, our BKG 
TABLE V

List OF Symbols USED IN THIS SECTION

\begin{tabular}{|l|l||l|l|}
\hline$C$ & Error-correcting code (vector space over $\left.\mathbb{Z}_{p}\right)$. & $\delta=(x-c)$ & Discretize biometric sample $x$ masked by $c$. \\
$n$ & Number of biometric features, as well as length of $C$. & $\gamma$ & Fuzzy commitment \\
$p$ & Alphabet size of $C$. $(p$ is a prime number larger $n)$. & $\mathrm{d}_{\text {rrange }} F_{i}$ & Upper bound of the range of DS $F_{i}$ \\
$l$ & Dimension of $C$ (size of the basis of $C)$. & $\min _{F_{i}}$, max $_{F_{i}}$ & Minimum and maximum values of $F_{i}$ \\
$w_{L}(\cdot), d_{L}(\cdot, \cdot)$ & Lee weight and Lee distance functions. & $\sigma_{i}$ & Standard deviation of $F_{i}$ \\
$z$ & System-wide public constant or user PIN/password. & $x_{i}$ & Instance of $F_{i}$ \\
$c=\left(c_{1}, \ldots, c_{n}\right)$ & Codeword from $C$. & $F_{i}$ & Biometric feature $i$ \\
$x=\left(x_{1}, \ldots, x_{n}\right)$ & Discretized biometric sample. & & \\
\end{tabular}

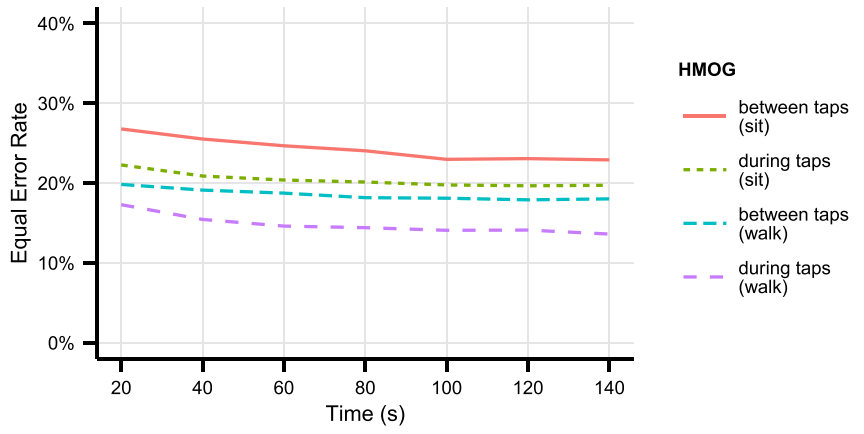

Fig. 9. Performance of HMOG features extracted during and between taps. $X$-axis shows authentication time in seconds.

construction represents features as symbols of an alphabet of arbitrary prime size $p$. Our BKG relies on Reed-Solomon error correcting codes [31] in Lee metric [32]. Notation used in this section is presented in Table $\mathrm{V}$.

Preliminaries: BKG uses biometric information to prevent unauthorized access to cryptographic keys; these keys can then be used, e.g., to encrypt/decrypt sensitive information. The process of protecting a key is referred to as committing, and the outcome of this process is a commitment. Given a commitment, the cryptographic key is reconstructed by decommiting (or opening) it, using information from a biometric signal. Informally, a BKG construction is secure if a key committed using a biometric signal $s$ can be opened only using a signal $s^{\prime} \approx s$, and both $s$ and $s^{\prime}$ are from the same user.

BKG techniques use error-correcting codes to address natural variations among different biometric samples from the same users. An error-correcting code is defined as a set $C$ of codewords. Typically, there are two functions associated with a code: encode $(\cdot)$ and decode $(\cdot)$. The former maps a message to a codeword; the latter-a possibly perturbed codeword to the original codeword. The decode $(\cdot)$ function is designed to maximize the probability of correct decoding.

\section{A. Our Construction}

1) Scaling and Discretization: BKG techniques work on discrete values, instead of real values. Therefore, the user first performs scaling and discretization of the feature vector representing her biometric. Each feature $F_{i}$ is assigned a discretization range $\left[0, d_{-}\right.$range $\left._{F_{i}}\right]$, where d_range $_{F_{i}} \in\{(p-1) / 2, \ldots, p-1\}$ is negatively correlated to the standard deviation $\sigma_{i}$ of $F_{i}$ (i.e., if $\sigma_{i}<\sigma_{j}$, then d_range $F_{i}>$ d_range $F_{j}$ ).

Let $x_{i}$ be an instance of $F_{i}$, and $\min _{F_{i}}$ and $\max _{F_{i}}$ be respectively the typical minimum and maximum value of $F_{i}$.
Discretization and scaling are performed as:

$$
\begin{aligned}
& \operatorname{DS}_{F_{i}}\left(x_{i}\right) \\
& \quad= \begin{cases}0 & x_{i}<\min _{F_{i}} \\
\left\lfloor\text { d_range }_{F_{i}} \cdot\left(\frac{x_{i}-\min _{F_{i}}}{\max _{F_{i}}-\min _{F_{i}}}\right)\right\rfloor & \min _{F_{i}} \leq x_{i} \leq \max _{F_{i}} \\
\text { d_range }_{F_{i}} & x_{i}>\max _{F_{i}}\end{cases}
\end{aligned}
$$

2) Committing a Key: To commit a cryptographic key using $n$ biometric features, the user selects a random codeword $c$ of length $n$ from $C \subset\left(\mathbb{Z}_{p}\right)^{n}$. The key is computed as $k=\operatorname{PRF}_{c}(z \mid 0)$, where PRF is a pseudorandom function family, $z$ is a system-wide public constant and "|" denotes string concatenation. (BKG can be augmented with a second authentication factor by setting $z$ to a user-provided password.) $c$ is then committed using the user's biometrics as discussed next. Let $x=\left(x_{1}, \ldots, x_{n}\right)$ be a scaled and discretized feature vector. The user computes $\delta=(x-c)=\left(x_{1}-c_{1}, \ldots, x_{n}-c_{n}\right)$ and publishes commitment $\gamma=\left(\operatorname{PRF}_{c}(z \mid 1), \delta\right)$.

The user computes $k$ from $\gamma$ and her biometric signals (and possibly a password $z$ ) as follows. She extracts biometric features from the signal, and encodes them as $y=\left(y_{1}, \ldots, y_{n}\right)$. Then, she computes $c^{\prime}=\operatorname{decode}(y-\delta)$. If $\operatorname{PRF}_{c^{\prime}}(z \mid 1)=$ $\operatorname{PRF}_{c}(z \mid 1)$, then $k=\operatorname{PRF}_{c^{\prime}}(z \mid 0)$ with overwhelming probability.

Asymptotic complexity of BKG key retrieval is dominated by one instance of Euclidean algorithm and one matrix-vector multiplication, both in $O\left(n^{2}\right)$ finite field operations in a field of size $p \geq n$. Security of our construction is analyzed in Appendix A.

3) Using Lee-Metric Decoding for BKG: Distance between feature vectors is defined using the Lee distance [32] - a discrete approximation of SM:

Definition 1 (Lee Weight): Let $p$ be an odd prime. The Lee weight of element $x \in \mathbb{Z}_{p}$ is defined as $w_{L}(x)=\min \left|x^{\prime}\right|$, for $x^{\prime} \equiv x \bmod p$. The Lee weight of vector $x=\left(x_{1}, \ldots, x_{n}\right) \in\left(\mathbb{Z}_{p}\right)^{n}$ is defined as the sum of Lee weights of its elements, i.e., $w_{L}(x)=\sum_{i=1}^{n} w_{L}\left(x_{i}\right)$.

Definition 2 (Lee Distance): The Lee distance of vectors $x, y \in \mathbb{Z}_{p}$ is the Lee weight of their difference, i.e., $d_{L}(x, y)=w_{L}(x-y)$.

In $\mathbb{Z}_{2}$, the Lee weight coincides with Hamming weight.

We used normalized generalized Reed-Solomon codes from [31], presented next, to implement the encode $(\cdot)$ and decode (.) functions.

Definition 3: Let $l \leq n$ and $n \leq p$. A linear $[n, l]$-code over $\mathbb{Z}_{p}$ is a l-dimensional vector subspace of $\left(\mathbb{Z}_{p}\right)^{n}$. A normalized Reed-Solomon $[n, l]$-code over $\mathbb{Z}_{p}$ is a linear $[n, l]$-code over 


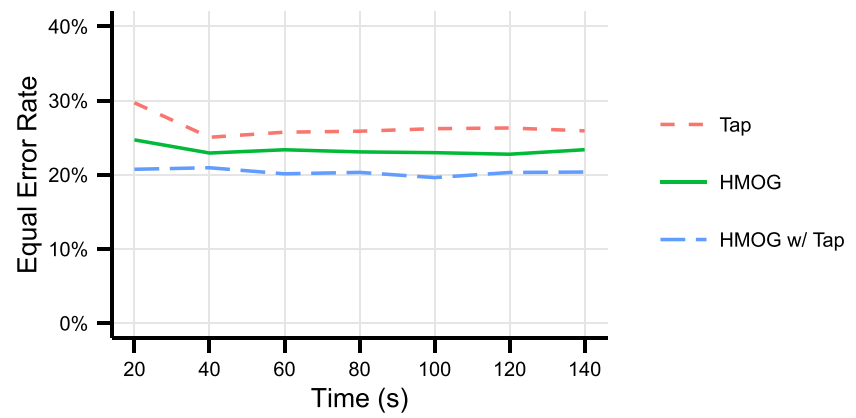

(a)

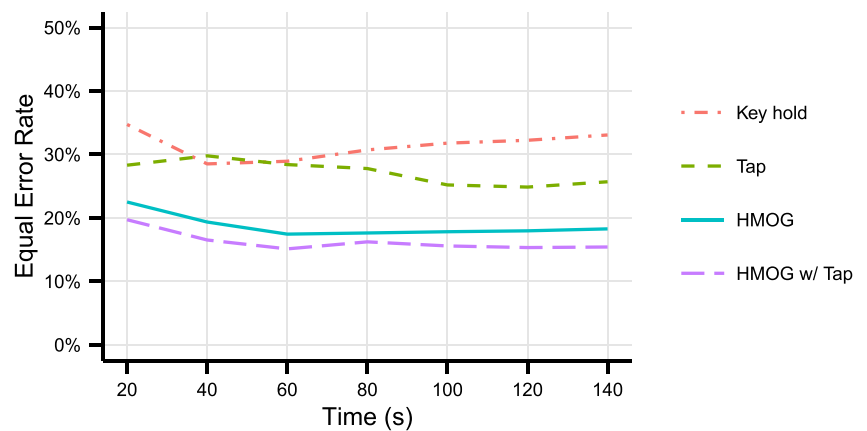

(b)

Fig. 10. EERs for BKG during sitting and walking. $X$-axis shows the authentication time in seconds. (a) EER for Sitting. (b) EER for Walking.

$\mathbb{Z}_{p}$ with parity-check matrix:

$$
H=\left(\begin{array}{cccc}
1 & 1 & \ldots & 1 \\
1 & 2 & \ldots & n \\
1 & 2^{2} & \ldots & n^{2} \\
& & \vdots & \\
1 & 2^{n-l-1} & \ldots & n^{n-l-1}
\end{array}\right)
$$

and generator matrix:

$$
G=\left(\begin{array}{cccc}
v_{1} & v_{2} & \ldots & v_{n} \\
v_{1} & 2 v_{2} & \ldots & n v_{n} \\
v_{1} & 2^{2} v_{2} & \ldots & n^{2} v_{n} \\
& & \vdots & \\
v_{1} & 2^{l-1} v_{2} & \ldots & n^{l-1} v_{n}
\end{array}\right)
$$

The rows of $G$ form a basis of the nullspace of $H^{T}$.

To obtain a random codeword from $C$, we select a $l$-tuple $m=\left(m_{1}, \ldots, m_{l}\right) \in\left(\mathbb{Z}_{p}\right)^{l}$ uniformly at random, and encode $m$ as $c=m G$. The Lee distance of $C$ is $2(n-l)$, so for any error $e=\left(e_{1}, \ldots, e_{n}\right)$ with $w_{L}(e)<n-l$, decode $(c+e)=c$.

\section{B. Evaluation of HMOG Features on $B K G$}

To evaluate HMOG features for $\mathrm{BKG}$, we determined their authentication accuracy and security against population attacks. We then compared our results with HMOG features to that of tap, key hold, and swipe features under the same metrics.

1) Biometric Accuracy: Figures 10(a) and 10(b) summarize the results of our experiments, performed on our 100-user dataset. We evaluated $\mathrm{BKG}$ using the features that performed best for authentication. We used 17 and 13 HMOG features in sitting and walking conditions, respectively (see Figure 3 ).

We ran experiments on four different feature subsets: (1) HMOG-only features; (2) 11 tap-only features; (3) 12 (sitting) and 8 (walking) key hold-only features; and (4) HMOG and 3 best-performing tap features. For both walking and sitting experiments, feature subset (4) provided the best results, i.e., $15 \%$ and $20 \%$ EER respectively, for both one-minute and two-minute scan lengths. For sitting experiment, key generation was not possible with (3), as the within-user variability of the biometrics signals was too high.

Šeděnka et al. [33] showed that Linear Discriminant Analysis (LDA) [34] improves BKG accuracy on desktop keystroke dynamics. However, HMOG, tap, and key hold features on a virtual keyboard did not benefit from LDA. Therefore we do not report BKG results with LDA for these features.

2) Security Against Population Attacks: EER computed via zero-effort attacks provides limited information on the security of a BKG scheme, because it does not take into account all the information readily available to the adversary. In particular, with BKG the adversary has access to: (1) the commitment $\gamma$; and (2) an approximation of the distribution of the user's biometric signals obtained from population data.

Access to $\gamma$ allows the adversary to test whether a particular feature vector decommits the key. The adversary can perform this test offline, i.e., with no restrictions on the number of attempts performed (within the limits of the available resources). Therefore, the hardness of "guessing" a user's feature vector given $\gamma$ is an upper bound on the security of a BKG scheme.

The adversary can use (2) to guess the user's feature vector more efficiently, under the assumption that biometric signals from different users are not completely independent. To this end, Ballard et al. [35] proposed the notion of guessing distance. It is defined as the logarithm of the number of guesses necessary to open a commitment using feature vectors from multiple impostors.

We instantiated guessing distance in our setting as follows. First, we built a commitment $\gamma_{i}=\left(\operatorname{PRF}_{c_{i}}(z \mid 1), \delta_{i}\right)$ from the feature vector of each user $i$. Then, we used the biometric sample from user $j$ to open all $\gamma_{i}$ such that $i \neq j$, and ranked users according to how many commitments they were able to open. Finally, for each user $i$ we select users $j \neq i$ following to this ranking, and determined how many attempts were required to open $\gamma_{i}$. Guessing distance was computed as the binary logarithm of this value. There might be users $i$ for which no feature vector from other users could open $\gamma_{i}$. We refer to the commitments of these users as non-guessed.

Table VI summarizes the results of our experiments for one minute scans. The lowest EER was achieved by combining HMOG features with the tap features selected by mRMR. Overall, HMOG outperformed tap features for biometric key generation. Nevertheless, our results show that most commitments can be guessed using population data.

3) Comparison of HMOG and Swipe Features: Because there is no previous work on BKG using touch-, accelerometer-, or gyroscope-based features, we compared 
TABLE VI

Security of BKG Based on HMOG, Tap, Key Hold, And Swipe Features

\begin{tabular}{llllll} 
Dataset & Features & EER & $\begin{array}{l}\text { Average } \\
\text { guessing } \\
\text { distance }\end{array}$ & $\begin{array}{l}\text { Non-guessed } \\
\text { commitments }\end{array}$ & $\begin{array}{l}\log _{2}(|C|) \\
\text { Our dataset-sitting }\end{array}$ \\
& HMOG & $23.4 \%$ & 2.8 & $2 \%$ & 19 \\
& TMOG with best 3 tap & $20.1 \%$ & 2.7 & $1 \%$ & $2 \%$ \\
\hline \multirow{3}{*}{ Our dataset-walking } & $25.7 \%$ & 1.6 & $2 \%$ & 27 \\
& HMOG & $17.4 \%$ & 2.9 & $5 \%$ & 33 \\
& Tap & $15.1 \%$ & 3.2 & $0 \%$ & 30 \\
\hline \multirow{2}{*}{ Serwadda et al. [21] } & Key hold & $28.4 \%$ & 1.9 & $0 \%$ & 10 \\
& caltrait orientation, verti- & $28.9 \%$ & 1.9 & $0 \%$ & 39
\end{tabular}

BKG on HMOG with BKG on touch features extracted from swipes (swipe features hereafter). For this purpose, we computed swipe features from the datasets of Serwadda et al. [21]. As in [21], we used the whole first session for computing commitments and ten swipes from the second session to perform impostor/genuine open attempts. Results are reported in Table VI.

We also performed experiments on the touch dataset of Frank et al. [6]. However, a large majority of the users could not reliably decommit their own keys. This was due to the large variance between the vectors used to build the commitment, and the one used to open it. Therefore, we did not include these results in this paper.

\section{ENERGy CONSUMPTION OF HMOG FEATURES}

We measured the energy consumption of two basic modules involved in extracting HMOG features: (1) sensors (i.e., accelerometer and gyroscope); and (2) feature computation (i.e., calculation of a HMOG feature from raw sensor readings). Our main finding from energy consumption analysis is that decreasing sensor sampling rate (from $100 \mathrm{~Hz}$ to as low as $16 \mathrm{~Hz}$ ) considerably reduced the energy overhead without impacting the authentication performance of HMOG features.

\section{A. Experiment Setup and Design}

We developed an Android application that collects and processes sensor data at different sampling rates. Our application allows us to selectively enable sensors and HMOG features. We extracted the best-performing 17 features for sitting (i.e., top-ranked 17 features in Figure 3(a) that were selected by $10-\mathrm{CV}$ ) and $13 \mathrm{HMOG}$ features for walking (i.e., top-ranked 13 features in Figure 3(b), selected by 10-CV). The union of these two feature sets resulted in 18 HMOG features. Because none of these feature were extracted from magnetometer, we did not measure its energy consumption.

Experiments were performed using a Samsung Galaxy S4 smartphone running Android 4.4. To obtain consistent and repeatable results, we terminated all other applications and all Google services on the smartphone. Additionally, we switched off WiFi, Bluetooth, and cellular radios. The screen was turned on during the experiments. Automatic brightness adjustments were disabled, and brightness was set to the lowest level. We used the Monsoon Power Monitor [36] to measure the phone's energy consumption.

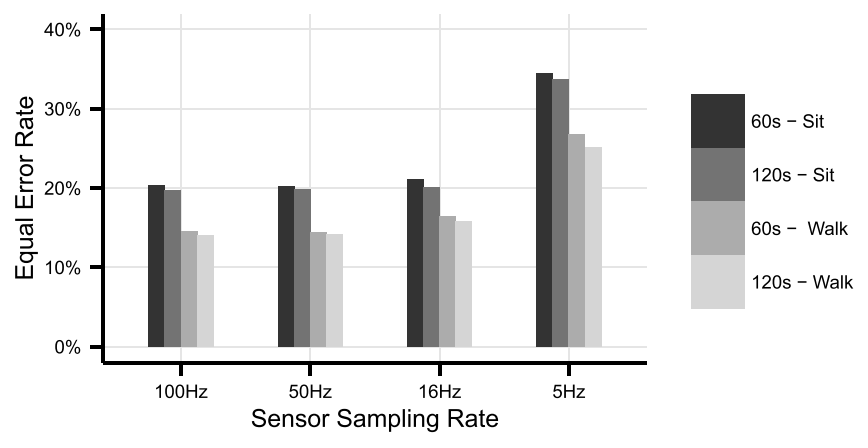

Fig. 11. Performance of HMOG features with different sensor sampling rates using SM verifier.

We performed the energy consumption experiments as follows. First, we measured baseline energy consumption by running our application with all sensors and features disabled. Then, we enabled accelerometer and gyroscope, and evaluated the corresponding energy consumption. Our application is designed to sample sensors at all supported frequencies. In the case of Galaxy S4, the available sampling rates are: $5 \mathrm{~Hz}$, $16 \mathrm{~Hz}, 50 \mathrm{~Hz}$, and $100 \mathrm{~Hz}$. We used authentication scan lengths of 60 and 120 seconds. Our results report the average and standard deviation of ten experiments in each setting. Finally, we quantified the energy overhead of computing $18 \mathrm{HMOG}$ features from sensor readings acquired during data collection.

Calculation of EERs at Lower Sampling Rates: We originally collected our data at $100 \mathrm{~Hz}$ sampling rate. In order to obtain the EERs for lower sampling rates, we used downsampling. For example, to simulate $16 \mathrm{~Hz}$ sampling rate, we choose every sixth sensor reading from the original sensor data. Then, using the downsampled data, we performed HMOG-based authentication with SM verifier for 60 - and 120-second scans using the same evaluation process as in Section IV.

\section{B. Energy Consumption of HMOG Authentication}

1) EERs vs. Energy Consumption: Figure 11 shows that EERs for $16 \mathrm{~Hz}$ sampling rate are comparable to those of $50 \mathrm{~Hz}$ and $100 \mathrm{~Hz}$ for both sitting and walking, while the EERs for $5 \mathrm{~Hz}$ are considerably worse than $16 \mathrm{~Hz}, 50 \mathrm{~Hz}$, and $100 \mathrm{~Hz}$.

On the other hand, Table VII shows that energy overhead over the baseline is low (between $6.2 \%$ and $7.9 \%$ ) for $5 \mathrm{~Hz}$ 
TABLE VII

ENERgy CONSUMPTION MEASUREMENT RESUlts

\begin{tabular}{c|llllll|} 
& \multicolumn{6}{|c}{60 Seconds Scan Length } \\
& & Baseline & $5 \mathrm{~Hz}$ & $16 \mathrm{~Hz}$ & $50 \mathrm{~Hz}$ & $100 \mathrm{~Hz}$ \\
\hline Energy & Mean & 42.7 & 45.5 & 46.1 & 48.4 & 51.5 \\
Consumption (J) & StdDev & 0.04 & 0.13 & 0.15 & 0.25 & 0.29 \\
\hline Overhead to Baseline & & N/A & $6.6 \%$ & $7.9 \%$ & $13.3 \%$ & $20.5 \%$
\end{tabular}

\begin{tabular}{c|llllll|} 
& \multicolumn{6}{|c|}{120 Seconds Scan Length } \\
& & Baseline & $5 \mathrm{~Hz}$ & $16 \mathrm{~Hz}$ & $50 \mathrm{~Hz}$ & $100 \mathrm{~Hz}$ \\
\hline Energy & Mean & 85.6 & 90.9 & 92.1 & 96.5 & 102.9 \\
Consumption (J) & StdDev & 0.17 & 0.21 & 0.23 & 0.30 & 0.36 \\
\hline Overhead to Baseline & & N/A & $6.2 \%$ & $7.6 \%$ & $12.8 \%$ & $20.1 \%$
\end{tabular}

and $16 \mathrm{~Hz}$ sampling rates and, in comparison, high (between $12.8 \%$ and $20.5 \%$ ) for $50 \mathrm{~Hz}$ and $100 \mathrm{~Hz}$.

Thus, during the active authentication with HMOG, we can choose $16 \mathrm{~Hz}$ instead of $100 \mathrm{~Hz}$ as the sensor sampling rate, which would lower the energy overhead of sensor data collection by about $60 \%$ without sacrificing EER.

2) Energy Consumption for Feature Computation: The energy overhead for computing 18 features is very low compared to energy overhead of sensor data collection. The energy consumption for computing all $18 \mathrm{HMOG}$ features is 0.08 joules, which corresponds to $0.19 \%$ overhead for the 60 -second and $0.1 \%$ for 120 -second scans. This low overhead can be attributed to the fact that HMOG features are time-domain features. (As suggested by previous research [37], [38], computing time-domain features consumes less energy than computing frequency-domain features.) Further, because HMOG feature computation involves simple arithmetic calculations, they can be processed very quickly by the smartphone's CPU (on average $37 \mathrm{~ms}$ per feature, in our case).

\section{RELATED WORK}

\section{A. Evolution of Continuous Authentication in Desktops and Mobile Phones}

The need to periodically authenticate the user after login, combined with the fact that behavioral biometric traits can be collected without interrupting the user, led to promising research in the area of continuous authentication. Early work in the field used keystroke dynamics [44]-[47] to authenticate desktop users. Later studies on desktop users demonstrated the feasibility of using a variety of behavioral traits, including mouse dynamics [25], soft-biometrics [48], hand movement [49], keyboard acoustics [50], screen fingerprints [51], language use [52], [53] and cognition during text production [27], [54]-[56].

Early studies in continuous authentication of mobile phone users focused on keystroke dynamics (see [57]-[61]), because these devices had a hardware keyboard to interface with the user. However, as mobile phones evolved into "smartphones", research in this area has been reshaped to leverage the multitude of available sensors on these devices (e.g., touchscreen, accelerometer, gyroscope, magnetometer, camera, and GPS). Two behavioral traits have been predominantly explored in the smartphone domain, (1) gait (see, e.g., [6], [21]), and (2) touchscreen interaction (see, e.g., [7], [62]). More recently, research has focused on leveraging multi-modal behaviors (e.g., [8], [63]).

\section{B. Continuous Authentication Using Taps}

Because HMOG features are collected during taps, we review existing work that uses tap activity to authenticate smartphone users. In Table VIII, we summarize the stateof-the-art in tap-based authentication, and highlight various aspects of each work, such as: (1) how the taps were collected-did the user compose free-text or type predefined fixed-text; (2) which body motion conditions (e.g., sitting and walking) were considered; (3) number of subjects (partitioned into owners and impostors, wherever appropriate); (4) how the verifier was trained; (5) how the authentication vector was created; and (6) the features used (e.g., motion-sensor, tap, or keystroke-based).

Among previous papers [8], [41], [43], which have used motion sensors for user authentication, Zheng et al. [41] used fixed pins while Gascon et al. [43] used fixed phrases. The only work that used free-text typing and also the only one to authenticate users under walking condition is the paper by Bo et al. [8]. Therefore, we believe that this is closest work to our paper, and highlight the differences between our paper and [8] as follows: (1) we performed experiments on a large-scale dataset containing 100 users (90 users qualified as genuine, and 93 or more as impostors), while [8] used only 10 genuine users and 50 impostors (on average) from a dataset of 100 subjects. Because the genuine population size in [8] is too small, it is difficult to assess how accurately the reported FARs/FRRs represent the achievable authentication error rates with movement-based features, given that the number of users is a critical factor in assessing the confidence on empirical error rates of biometric systems [64]; (2) we introduced and evaluated a wide range of movement features, while [8] used only two (i.e., mean magnitude of acceleration and mean magnitude of angular velocity, during a gesture). Our results clearly reveal that certain types of movement features (e.g., resistance) perform better than others (e.g., stability), while [8] does not distinguish between different types of movement features; (3) our evaluation is comprehensive and includes detailed comparison and fusion with additional features such as touchscreen tap and keystroke. This allowed us to report how fusion with different types of features impacted authentication and BKG performance. In contrast, [8] do not compare different types of features; and (4) HMOG features performed well in both sitting and walking condition, while [8] had resorted to gait features for authentication during walking.

\section{Biometric Key Generation}

To our knowledge, there is no previous work on BKG on smartphones. Here, we review some important work related to BKG in general.

Introduced by Juels and Wattenberg [30], BKG implemented via fuzzy commitments uses error correcting codes to construct cryptographic keys from noisy information. Features are extracted from raw signals (e.g., minutiae from fingerprint images); then, each feature is encoded using a 
TABLE VIII

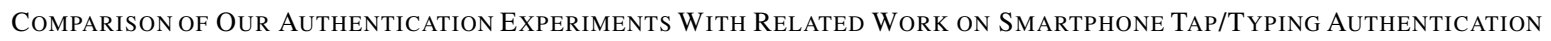

\begin{tabular}{|c|c|c|c|c|c|c|c|c|c|c|c|c|}
\hline Work & Condition & Free text & Motion-based features & Tap features & $\begin{array}{l}\text { Keystroke } \\
\text { features }\end{array}$ & $\begin{array}{l}\text { Authentication } \\
\text { vector }\end{array}$ & $\begin{array}{l}\text { \# of } \\
\text { owners }\end{array}$ & $\begin{array}{l}\text { Avg. \# of } \\
\text { impostors } \\
\text { per owner }\end{array}$ & Verifier & $\begin{array}{l}\text { Training } \\
\text { data source }\end{array}$ & Best FAR & Best FRR \\
\hline Trojahn et al. [39] & sit & $x$ & $x$ & $\begin{array}{l}\text { pressure, } \\
\text { contact size }\end{array}$ & digraph & avg. of 7 samples & 35 & 34 & ANN & unknown & $9.53 \%$ & $5.88 \%$ \\
\hline Li et al. [40] & regular sma & hone usage & $x$ & $\begin{array}{l}\text { pressure, touch } \\
\text { area, duration }\end{array}$ & $x$ & avg. of 2-20 gestures & 28 & up to 47 & $\begin{array}{l}\text { SVM (Gaus- } \\
\text { sian kernel) }\end{array}$ & $\begin{array}{l}\text { owner \& } \\
\text { impostor }\end{array}$ & \multicolumn{2}{|c|}{ not reported for taps } \\
\hline Zheng et al. [41] & sit & $x$ & $\begin{array}{l}\min , \max \text { and mean of accele- } \\
\text { ration and angular velocity at } \\
\text { press, release of each PIN digit }\end{array}$ & $\begin{array}{l}\text { pressure, contact } \\
\text { size (both at } \\
\text { press and release) }\end{array}$ & $\begin{array}{l}\text { key hold, } \\
\text { key interval }\end{array}$ & each tap & 80 & 79 & $\begin{array}{l}\text { dissimilarity } \\
\text { score }\end{array}$ & owner & \multicolumn{2}{|c|}{$\mathrm{EER}=3.65 \%$} \\
\hline Feng et al. [42] & sit & $x$ & 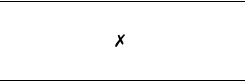 & pressure & $\begin{array}{l}\text { key hold, } \\
\text { key interval }\end{array}$ & 5-60 char. window & 40 & 39 & $\begin{array}{l}\text { decision tree, } \\
\text { Bayesian } \\
\text { networks, } \\
\text { random forest }\end{array}$ & $\begin{array}{l}\text { owner \& } \\
\text { impostor }\end{array}$ & \multicolumn{2}{|l|}{$\mathrm{EER}=1 \%$} \\
\hline Gascon et al. [43] & sit & $x$ & $\begin{array}{l}\text { accelerometer, gyroscope, and } \\
\text { orientation features extracted } \\
\text { during typing burst }\end{array}$ & $x$ & $x$ & $\begin{array}{l}\text { features extracted } \\
\text { from time window }\end{array}$ & 12 & 303 & linear SVM & $\begin{array}{l}\text { owner \& } \\
\text { impostor }\end{array}$ & $\begin{array}{l}1 \% \text { (4 genuine } \\
\text { users) }\end{array}$ & $\begin{array}{l}8 \% \text { ( } 4 \text { gen- } \\
\text { uine users) }\end{array}$ \\
\hline Bo et al. [8] & sit, walk & $\checkmark$ & $\begin{array}{l}\text { mean magnitude of acceleration } \\
\text { and angular velocity during tap }\end{array}$ & $\begin{array}{l}\text { coordinate, pres- } \\
\text { sure, duration }\end{array}$ & $x$ & $\begin{array}{l}\text { each gesture, } \\
\text { judgement after } \\
1-13 \text { gestures }\end{array}$ & 10 & 50 & SVM & $\begin{array}{l}\text { owner only, } \\
\text { and owner \& } \\
\text { impostor }\end{array}$ & $\begin{array}{l}0 \% \text { when trained } \\
\text { with owner \& } \\
\text { impostor data, } 24.99 \% \\
\text { with owner data }\end{array}$ & $\begin{array}{l}0 \% \text { when } \\
\text { trained with } \\
\text { owner data }\end{array}$ \\
\hline This work & sit, walk & $\checkmark$ & $\begin{array}{l}60 \text { resistance and } 36 \text { stability } \\
\text { features extracted from tap, } \\
\text { using accelerometer, gyroscope } \\
\text { and magnetometer }\end{array}$ & $\begin{array}{l}\text { contact size }(9 \\
\text { f.), duration, } \\
\text { velocity between } \\
\text { two taps }\end{array}$ & $\begin{array}{l}\text { key hold, } \\
\text { digraph }\end{array}$ & $\begin{array}{l}\text { taps averaged } \\
\text { in time window } \\
(20,40,60,80 \\
100,120,140 \mathrm{~s} .)\end{array}$ & 90 & $\begin{array}{l}99 \text { sit } \\
93 \text { walk }\end{array}$ & $\begin{array}{l}\text { SM, SE, } \\
\text { 1-class SVM }\end{array}$ & owner & \multicolumn{2}{|c|}{$\mathrm{EER}=7.16 \%$} \\
\hline
\end{tabular}

single bit. Cryptographic keys are committed using features; subsequently, commitments are opened using biometric signals from the same users. Error-correcting techniques are applied to noisy biometric information in order to cope with within-user variance.

Ballard et al. [35] provided a formal framework for analyzing the security of a BKG scheme, and argued that BKG should enjoy biometric privacy (i.e., biometric signals cannot be reconstructed from biometric keys) and key randomness (i.e., keys look random given their commitment). They also formalized adversarial knowledge of the biometric by introducing guessing distance-the logarithm of the number of guesses necessary to open a commitment using feature vectors from multiple impostors.

\section{Energy Consumption Analysis}

Bo et al. [8] showed that energy consumption can be reduced by selectively turning off motion sensors based on two factors: (1) the sensitivity of the app being used-non-sensitive applications, such as games, require no authentication; and (2) the probability that the smartphone is handed to another user. This probability is calculated using historical smartphone usage data. In their experiments, Bo et al. were able to turn off the sensors $30-90 \%$ of the time, while maintaining reasonable authentication performance. However, they did not report how they performed energy consumption measurements, nor listed the energy consumptions associated with determining if the phone was being held by its owner or handed to another user.

Feng et al. [65] introduced TIPS — a continuous user authentication technique that relies on touch features exclusively. By collecting energy usage data, the authors reported average energy consumption of $88 \mathrm{~mW}$, which corresponds to less than $6.2 \%$ overhead. Like [8], Feng et al. [65] also do not describe how energy measurements were performed.

Khan et al. presented Itus [66], a framework that helps Android application developers to deploy various continuous authentication mechanisms. Energy evaluation was performed using PowerTutor [67] —an Android application that reports energy measurements performed by the smartphone. Overall energy overhead of the tested continuous authentication techniques varied between $1.2 \%$ and $6.2 \%$.

Compared to previous research, our work provides a more complete picture of energy overhead of continuous authentication using HMOG. In fact, we highlighted the tradeoffs of energy usage for different sensor sampling rates and authentication scan lengths, versus authentication accuracy. In comparison to our work, existing literature did not analyze fine-grained energy consumption brought by individual components such as motion sensors. To our knowledge, we are the first to report the relationship between sensor sampling rates and continuous authentication accuracy.

\section{CONCLUSION AND Future WORK}

In this paper, we introduced HMOG, a set of behavioral biometric features for continuous authentication of smartphone users. We evaluated HMOG from three perspectives-continuous authentication, BKG, and energy consumption. Our evaluation was performed on multi-session data collected from 100 subjects under two motion conditions (i.e., sitting and walking). Results of our evaluation can be summarized as follows. By combining HMOG with tap features, we achieved $8.53 \%$ authentication EER during walking and $11.41 \%$ during sitting, which is lower than the EERs achieved individually with tap or HMOG features. Further, by fusing HMOG, tap and keystroke dynamic features, we achieved the lowest EERs $(7.16 \%$ in walking and $10.05 \%$ in sitting). Our results demonstrate that HMOG is well suited for continuous authentication of smartphone users. In fact, HMOG improves the performance of taps and keystroke dynamic features, especially during walking-a common smartphone usage scenario. For BKG, HMOG features provide lower EER (17.4\%) compared to tap $(25.7 \%)$ and swipe features (34.2\%). Moreover, fusion of HMOG with tap features provide the best performance, with $15.1 \%$ EER. Additionally, the energy overhead of sample collection and feature extraction is small (less than $8 \%$ energy overhead when sensors were sampled at $16 \mathrm{~Hz}$ ). This makes HMOG well suited for energy-constrained devices such as smartphones.

As future work, we plan to investigate how HMOG features perform under stringent constraints such as: (a) walking at higher speeds; (b) using the smartphone in different weather conditions; and (c) using applications that do not involve typing (e.g., browsing a map). Another research question of interest is cross-device interoperability, i.e., how and to what extent can a user's behavioral biometric collected on a desktop 
(e.g., keystroke dynamics) be leveraged with HMOG features to authenticate the user on a smartphone (and vice versa).

\section{APPENDIX A \\ SECURITy ANALYSis OF OUR BKG SCHEME}

We prove that our BKG technique meets the requirements from [35] - namely, that cryptographic keys are indistinguishable from random given the commitment (key randomness), and that given a cryptographic key and a commitment, no useful information about the biometric can be reconstructed (biometric privacy). We assume that the biometric is modeled by an unpredictable function. This captures the idea that a user's biometric is difficult to guess. Informally, an unpredictable function $f(\cdot)$ is a function for which no efficient adversary can predict $f\left(x^{*}\right)$ given $f\left(x_{i}\right)$ for various $x_{i} \neq x^{*}$. Formally:

Definition 4: A function family $(\mathcal{C}, D, R, F)$ for $\left\{f_{c}(\cdot)\right.$ : $D \rightarrow R\}_{c \leftarrow \mathcal{C}}$ is unpredictable if for any efficient algorithm $\mathcal{A}$ and auxiliary information $z$ we have:

$$
\operatorname{Pr}\left[\left(x^{*}, f_{c}\left(x^{*}\right) \leftarrow \mathcal{A}^{f_{c}(\cdot)}(z) \text { and } x^{*} \notin Q\right] \leq \operatorname{negl}(\kappa)\right.
$$

where $Q$ is the set of queries from $\mathcal{A}, \kappa$ is the security parameter and negl(.) is a negligible function.

In order to define security of biometric key generation systems, Ballard et al. [35] introduced the notions of Key Randomness (REQ-KR), Weak Biometric Privacy (REQ-WBP) and Strong Biometric Privacy (REQ-SBP). We formalize the notion of key randomness by defining Experiment $\operatorname{IND}_{-K_{\mathcal{A}}}(\kappa)$ :

Experiment $\mathrm{IND}_{-\mathrm{KR}_{\mathcal{A}}}(\kappa)$ :

1) $\mathcal{A}$ is provided with a challenge $\left(\operatorname{PRF}_{c_{i}}(z \mid 1), \delta\right)$, $k_{b}$ and $z$, where $k_{0}=\operatorname{PRF}_{c_{i}}(z \mid 0)$ and $k_{1} \leftarrow_{R}\{0,1\}^{\kappa}$ for a bit $b \leftarrow_{R}\{0,1\}$, corresponding to user $i$.

2) $\mathcal{A}$ is allowed to obtain biometric information $x_{j}$ for arbitrary users $j$ such that $j \neq i$.

3) $\mathcal{A}$ outputs a bit $b^{\prime}$ as its guess for $b$. The experiment outputs 1 if $b=b^{\prime}$, and 0 otherwise.

Definition 5: We say that a biometric key generation scheme has the Key Randomness property if there exist a negligible function negl(.) such that for any $P P T \mathcal{A}$, $\operatorname{Pr}\left[\operatorname{IND}-\operatorname{KR}_{\mathcal{A}}(\kappa)=1\right] \leq 1 / 2+\operatorname{negl}(\kappa)$.

Theorem 1: Assuming that the PRF is a pseudo-random function family and that biometric $x=\left(x_{1}, \ldots, x_{n}\right)$ is unpredictable, our Fuzzy Commitment scheme has the Key Randomness property.

Proof of Theorem 1 (Sketch): Because $c=x-\delta$, and $x$ is assumed to be unpredictable, $c$ is unpredictable given $\delta$. We now show that any PPT adversary $\mathcal{A}$ that has advantage $1 / 2+\Delta(\kappa)$ to win the IND-KR $\mathrm{KR}_{\mathcal{A}}(\kappa)$ experiment can be used to construct a distinguisher $\mathcal{D}$ that has similar advantage in distinguishing PRF from a family of uniformly distributed random functions.

$\mathcal{D}$ is given access to oracle $O(\cdot)$ that selects a random codeword $c$ and a random bit $b$, and responds to a query $q$ with random (consistent) values if $b=1$, and with $\operatorname{PRF}_{c}(q)$ if $b=0$. D selects a random $z$, a codeword $c^{\prime}$ and a feature vector $x^{\prime}$, and sets $\delta^{\prime}=x^{\prime}-c^{\prime}$. Then $\mathcal{D}$ sends $\gamma^{\prime}=\left(O(z \mid 1), \delta^{\prime}\right)$ and $O(z \mid 0)$ to $\mathcal{A}$.
If $b=0$, then pair $\left(\gamma^{\prime}, \operatorname{PRF}_{c^{\prime}}(z \mid 0)\right)$ is indistinguishable from $\left(\left(\operatorname{PRF}_{c}(z \mid 1), \delta\right), \operatorname{PRF}_{c}(z \mid 0)\right)$, because $\delta$ and $\delta^{\prime}$ follow the same distribution, $c$ and $c^{\prime}$ are unpredictable given $\delta$ and thus both $\operatorname{PRF}_{c}(\cdot)$ and $\operatorname{PRF}_{c^{\prime}}(\cdot)$ are indistinguishable from random. If $b=1$, then $O(\cdot)$ is a random oracle, so $\left(\gamma^{\prime}, O(z)\right)$ is indistinguishable from pair $\left(\left(\operatorname{PRF}_{c}(z \mid 1), \delta\right), \operatorname{PRF}_{c}(z \mid 0)\right): c$ is unpredictable given $\delta$, therefore $\operatorname{PRF}_{c}(\cdot)$ is indistinguishable from random.

Eventually, $\mathcal{A}$ outputs $b^{\prime}$, and $\mathcal{D}$ outputs $b^{\prime}$ as its guess. It is easy to see that $\mathcal{D}$ wins iff $\mathcal{A}$ wins, so $\mathcal{D}$ is correct with probability $1 / 2+\Delta(\kappa)$. Therefore, $\Delta(\cdot)$ must be a negligible function.

REQ-WBP states that the adversary learns no useful information about a biometric signal from the commitment and the auxiliary information, while REQ-SBP states that the adversary learns no useful information about the biometric given auxiliary information, the commitment and the key. For our BKG algorithms, REQ-SBP implies REQ-WBP. In fact, $\operatorname{PRF}_{c}(z \mid 1)$ (which is part of the commitment) is known to the adversary, and therefore $k=\operatorname{PRF}_{c}(z \mid 0)$ does not reveal any additional information. From the unpredictability of $x$, it follows that the output of $\mathrm{PRF}_{c}$ does not reveal $c$, so $\mathrm{PRF}_{c}(z \mid 1)$ and $k$ do not disclose information about $x$.

\section{ACKNOWLEDGMENT}

The views, findings, recommendations, and conclusions contained herein are those of the authors and should not be interpreted as necessarily representing the official policies or endorsements, either expressed or implied, of the sponsoring agencies or the U.S. Government.

Computing and storage resources were provided in part by the Research Program "Projects of Large Infrastructure for Research, Development, and Innovations” (LM2010005).

\section{REFERENCES}

[1] Data Genetics: Pin Analysis. [Online]. Available: http://www. datagenetics.com/blog/september32012/, accessed Jan. 8, 2015.

[2] SRLabs: Spoofing Fingerprints. [Online]. Available: https://srlabs.de/ spoofing-fingerprints/, accessed Jan. 8, 2015.

[3] A. J. Aviv, K. Gibson, E. Mossop, M. Blaze, and J. M. Smith, "Smudge attacks on smartphone touch screens," in Proc. 4th USENIX Workshop Offensive Technol. (WOOT), Washington, DC, USA, Aug. 2010, pp. $1-10$.

[4] Y. Xu, J. Heinly, A. M. White, F. Monrose, and J.-M. Frahm, "Seeing double: Reconstructing obscured typed input from repeated compromising reflections," in Proc. ACM SIGSAC Conf. Comput. Commun. Secur. (CCS), Berlin, Germany, Nov. 2013, pp. 1063-1074.

[5] D. Shukla, R. Kumar, A. Serwadda, and V. V. Phoha, "Beware, your hands reveal your secrets!" in Proc. ACM SIGSAC Conf. Comput. Commun. Secur., Scottsdale, AZ, USA, Nov. 2014, pp. 904-917.

[6] M. Frank, R. Biedert, E. Ma, I. Martinovic, and D. Song, "Touchalytics: On the applicability of touchscreen input as a behavioral biometric for continuous authentication," IEEE Trans. Inf. Forensics Security, vol. 8, no. 1, pp. 136-148, Jan. 2013.

[7] M. O. Derawi, C. Nickel, P. Bours, and C. Busch, "Unobtrusive userauthentication on mobile phones using biometric gait recognition," in Proc. 6th Int. Conf. Intell. Inf. Hiding Multimedia Signal Process., Oct. 2010, pp. 306-311.

[8] C. Bo, L. Zhang, X.-Y. Li, Q. Huang, and Y. Wang, "SilentSense: Silent user identification via touch and movement behavioral biometrics," in Proc. 19th Annu. Int. Conf. MobiCom, 2013, pp. 187-190.

[9] H. Lu, A. J. B. Brush, B. Priyantha, A. K. Karlson, and J. Liu, "SpeakerSense: Energy efficient unobtrusive speaker identification on mobile phones," in Proc. 9th Int. Conf. Pervasive Comput. (Pervasive), San Francisco, CA, USA, Jun. 2011, pp. 188-205. 
[10] E. Shi, Y. Niu, M. Jakobsson, and R. Chow, "Implicit authentication through learning user behavior," in Proc. 13th Int. Conf. Inf. Secur., 2010, pp. 99-113.

[11] J. R. Napier, "The prehensile movements of the human hand," J. Bone Joint Surgery, vol. 38-B, no. 4, pp. 902-913, 1956.

[12] A. K. Karlson, B. B. Bederson, and J. L. Contreras-Vidal, "Studies in one-handed mobile design: Habit, desire and agility," in Proc. 4th ERCIM Workshop User Interfaces All (UI4ALL), 2006, pp. 1-10.

[13] S. Azenkot and S. Zhai, "Touch behavior with different postures on soft smartphone keyboards," in Proc. 14th Int. Conf. MobileHCI, 2012, pp. 251-260.

[14] J. O. Wobbrock, B. A. Myers, and H. H. Aung, "The performance of hand postures in front- and back-of-device interaction for mobile computing," Int. J. Human-Comput. Stud., vol. 66, no. 12, pp. 857-875, 2008.

[15] I. M. Fiebert, K. E. Roach, J. W. Fromdahl, J. D. Moyer, and F. F. Pfeiffer, "Relationship between hand size, grip strength and dynamometer position in women," J. Back Musculoskeletal Rehabil., vol. 10, no. 3, pp. 137-142, 1998 .

[16] S. Chatterjee and B. J. Chowdhuri, "Comparison of grip strength and isometric endurance between the right and left hands of men and their relationship with age and other physical parameters," J. Human Ergol., vol. 20, no. 1, pp. 41-50, 1991.

[17] K.-E. Kim et al., "Hand grip pattern recognition for mobile user interfaces," in Proc. 18th Conf. Innov. Appl. Artif. Intell. (IAAI), vol. 2. 2006, pp. 1789-1794.

[18] S. Mizobuchi, M. Chignell, and D. Newton, "Mobile text entry: Relationship between walking speed and text input task difficulty," in Proc. 7th Int. Conf. Human Comput. Interact. Mobile Devices Services, 2005, pp. $122-128$.

[19] Q. Yang et al., "A multimodal data set for evaluating continuous authentication performance in smartphones," in Proc. 12th ACM Conf. SenSys, 2014, pp. 358-359.

[20] K. S. Killourhy and R. A. Maxion, "Comparing anomaly-detection algorithms for keystroke dynamics," in Proc. IEEE/IFIP Int. Conf. Dependable Syst. Netw., Jun./Jul. 2009, pp. 125-134.

[21] A. Serwadda, V. V. Phoha, and Z. Wang, "Which verifiers work? A benchmark evaluation of touch-based authentication algorithms," in Proc. 6th Int. Conf. BTAS, Sep./Oct. 2013, pp. 1-8.

[22] M. Blanton and P. Gasti, "Secure and efficient protocols for iris and fingerprint identification," in Proc. ESORICS, 2011, pp. 190-209.

[23] S. Govindarajan, P. Gasti, and K. S. Balagani, "Secure privacypreserving protocols for outsourcing continuous authentication of smartphone users with touch data," in Proc. IEEE 6th Int. Conf. BTAS, Sep./Oct. 2013, pp. 1-8.

[24] C.-C. Chang and C.-J. Lin, "LIBSVM: A library for support vector machines," ACM Trans. Intell. Syst. Technol., vol. 2, no. 3, 2011, Art. ID 27.

[25] C. Shen, Z. Cai, X. Guan, Y. Du, and R. A. Maxion, "User authentication through mouse dynamics," IEEE Trans. Inf. Forensics Security, vol. 8, no. 1, pp. 16-30, Jan. 2013.

[26] A. Ross and A. Jain, "Information fusion in biometrics," Pattern Recognit. Lett., vol. 24, no. 13, pp. 2115-2125, 2003.

[27] H. Locklear et al., "Continuous authentication with cognition-centric text production and revision features," in Proc. IEEE IJCB, Sep./Oct. 2014, pp. $1-8$.

[28] R. O. Duda, P. E. Hart, and D. G. Stork, Pattern Classification, 2nd ed. Hoboken, NJ, USA: Wiley, 2001

[29] H. Peng, F. Long, and C. Ding, "Feature selection based on mutual information criteria of max-dependency, max-relevance, and minredundancy," IEEE Trans. Pattern Anal. Mach. Intell., vol. 27, no. 8 , pp. 1226-1238, Aug. 2005.

[30] A. Juels and M. Wattenberg, "A fuzzy commitment scheme," in Proc. 6th ACM Conf. CCS, 1999, pp. 28-36.

[31] R. M. Roth, Introduction to Coding Theory. Cambridge, U.K.: Cambridge Univ. Press, 2006.

[32] C. Lee, "Some properties of nonbinary error-correcting codes," IRE Trans. Inf. Theory, vol. 4, no. 2, pp. 77-82, Jun. 1958.

[33] J. Šeděnka, V. Phoha, K. S. Balagani, and P. Gasti, "Privacy-preserving population-enhanced biometric key generation from free-text keystroke dynamics," in Proc. IEEE IJCB, Sep./Oct. 2014, pp. 1-8.

[34] R. A. Fisher, "The use of multiple measurements in taxonomic problems," Ann. Eugenics, vol. 7, no. 2, pp. 179-188, 1936.

[35] L. Ballard, S. Kamara, and M. K. Reiter, "The practical subtleties of biometric key generation," in Proc. USENIX 17th Conf. Secur. Symp., 2008, pp. 61-74.
[36] Monsoon Power Monitor. [Online]. Available: http://www.msoon.com/ LabEquipment/PowerMonitor/, accessed Nov. 12, 2014.

[37] A. Krause et al., "Trading off prediction accuracy and power consumption for context-aware wearable computing," in Proc. IEEE 9th Int. Symp. Wearable Comput., Oct. 2005, pp. 20-26.

[38] Z. Yan, V. Subbaraju, D. Chakraborty, A. Misra, and K. Aberer, "Energy-efficient continuous activity recognition on mobile phones: An activity-adaptive approach," in Proc. 16th Int. Symp. Wearable Comput., Jun. 2012, pp. 17-24.

[39] M. Trojahn and F. Ortmeier, "Biometric authentication through a virtual keyboard for smartphones," Int. J. Comput. Sci. Inf. Technol., vol. 4, no. 5, pp. 1-12, 2012.

[40] L. Li, X. Zhao, and G. Xue, "Unobservable re-authentication for smartphones," in Proc. NDSS, 2013, pp. 1-16.

[41] N. Zheng, K. Bai, H. Huang, and H. Wang, "You are how you touch: User verification on smartphones via tapping behaviors," Dept. Comput. Sci., College William Mary, Williamsburg, VA, USA, Tech. Rep. WM-CS-2012-06, 2012.

[42] T. Feng, X. Zhao, B. Carbunar, and W. Shi, "Continuous mobile authentication using virtual key typing biometrics," in Proc. 12th IEEE Int. Conf. TRUSTCOM, Jul. 2013, pp. 1547-1552.

[43] H. Gascon, S. Uellenbeck, C. Wolf, and K. Rieck, "Continuous authentication on mobile devices by analysis of typing motion behavior," in Proc. Sicherheit, 2014, pp. 1-12.

[44] D. Gunetti and C. Picardi, "Keystroke analysis of free text," $A C M$ Trans. Inf. Syst. Secur., vol. 8, no. 3, pp. 312-347, Aug. 2005. [Online]. Available: http://doi.acm.org/10.1145/1085126.1085129

[45] P. Dowland, S. Furnell, and M. Papadaki, "Keystroke analysis as a method of advanced user authentication and response," in Proc. IFIP TC11 17th Int. Conf. Inf. Secur., Vis. Perspect. (SEC), Deventer, The Netherlands, 2002, pp. 215-226. [Online]. Available: http://dl.acm. org/citation $. \mathrm{cfm} ? \mathrm{id}=647185.719834$

[46] F. Monrose and A. Rubin, "Authentication via keystroke dynamics," in Proc. 4th ACM Conf. Comput. Commun. Secur. (CCS), 1997, pp. 48-56. [Online]. Available: http://doi.acm.org/10.1145/266420.266434

[47] P. S. Dowland, S. M. Furnell, and M. Papadaki, "Keystroke analysis as a method of advanced user authentication and response," in Security in the Information Society. USA: Springer, 2002, pp. 215-226.

[48] K. Niinuma, U. Park, and A. K. Jain, "Soft biometric traits for continuous user authentication," IEEE Trans. Inf. Forensics Security, vol. 5, no. 4, pp. 771-780, Dec. 2010.

[49] J. Roth, X. Liu, and D. Metaxas, "On continuous user authentication via typing behavior," IEEE Trans. Image Process., vol. 23, no. 10, pp. 4611-4624, Oct. 2014

[50] J. Roth, X. Liu, A. Ross, and D. Metaxas, "Investigating the discriminative power of keystroke sound," IEEE Trans. Inf. Forensics Security, vol. 10, no. 2, pp. 333-345, Feb. 2015.

[51] M. E. Fathy, V. M. Patel, T. Yeh, Y. Zhang, R. Chellappa, and L. S. Davis, "Screen-based active user authentication," Pattern Recognit. Lett., vol. 42, pp. 122-127, Jun. 2014.

[52] A. Stolerman, A. Fridman, R. Greenstadt, P. Brennan, and P. Juola, "Active linguistic authentication revisited: Real-time stylometric evaluation towards multi-modal decision fusion," in Proc. IFIP WG, vol. 11. 2014, pp. 1-11.

[53] N. Pokhriyal, I. Nwogu, and V. Govindaraju, "Use of language as a cognitive biometric trait," in Proc. IEEE Int. Joint Conf. Biometrics (IJCB), Sep./Oct. 2014, pp. 1-8.

[54] J. V. Monaco, N. Bakelman, S.-H. Cha, and C. C. Tappert, "Developing a keystroke biometric system for continual authentication of computer users," in Proc. Eur. Intell. Secur. Inform. Conf., Aug. 2012, pp. $210-216$.

[55] J. C. Stewart, J. V. Monaco, S.-H. Cha, and C. C. Tappert, "An investigation of keystroke and stylometry traits for authenticating online test takers," in Proc. Int. Joint Conf. Biometrics, Oct. 2011, pp. $1-7$.

[56] J. V. Monaco, J. C. Stewart, S.-H. Cha, and C. C. Tappert, "Behavioral biometric verification of student identity in online course assessment and authentication of authors in literary works," in Proc. IEEE 6th Int. Conf. Biometrics, Theory, Appl., Syst., Sep./Oct. 2013, pp. 1-8.

[57] N. L. Clarke and S. M. Furnell, "Authenticating mobile phone users using keystroke analysis," Int. J. Inf. Secur., vol. 6, no. 1, pp. 1-14, 2007.

[58] N. L. Clarke and S. M. Furnell, "Advanced user authentication for mobile devices," Comput. Secur., vol. 26, no. 2, pp. 109-119, 2007.

[59] A. Buchoux and N. L. Clarke, "Deployment of keystroke analysis on a smartphone," in Proc. Austral. Inf. Secur. Manage. Conf., 2008, p. 48. 
[60] E. Maiorana, P. Campisi, N. González-Carballo, and A. Neri, "Keystroke dynamics authentication for mobile phones," in Proc. ACM Symp. Appl. Comput., 2011, pp. 21-26.

[61] P. Campisi, E. Maiorana, M. Lo Bosco, and A. Neri, "User authentication using keystroke dynamics for cellular phones," IET Signal Process., vol. 3, no. 4, pp. 333-341, Jul. 2009.

[62] E. Vildjiounaite et al., "Unobtrusive multimodal biometrics for ensuring privacy and information security with personal devices," in Proc. 4th Int. Conf. Pervasive, 2006, pp. 187-201.

[63] W. Shi, J. Yang, Y. Jiang, F. Yang, and Y. Xiong, "SenGuard: Passive user identification on smartphones using multiple sensors," in Proc. IEEE 7th Int. Conf. Wireless Mobile Comput., Netw., Commun. (WiMob), Oct. 2011, pp. 141-148.

[64] S. C. Dass, Y. Zhu, and A. K. Jain, "Validating a biometric authentication system: Sample size requirements," IEEE Trans. Pattern Anal. Mach. Intell., vol. 28, no. 12, pp. 1902-1913, Dec. 2006.

[65] T. Feng, J. Yang, Z. Yan, E. M. Tapia, and W. Shi, "TIPS: Contextaware implicit user identification using touch screen in uncontrolled environments," in Proc. 15th Workshop Mobile Comput. Syst. Appl., 2014, Art. ID 9.

[66] H. Khan, A. Atwater, and U. Hengartner, "Itus: An implicit authentication framework for Android," in Proc. 20th Annu. Int. Conf. MobiCom, 2014, pp. 507-518.

[67] PowerTutor. [Online]. Available: http://ziyang.eecs.umich.edu/projects/ powertutor/, accessed Nov. 18, 2014.

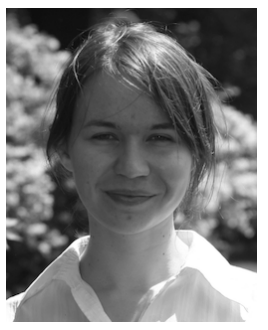

Zdeňka Sitová received the B.Sc. and M.Sc. degrees in computer science from Masaryk University, Brno, Czech Republic. She is currently a Bioinformatician with the Mendel Centre for Plant Genomics and Proteomics, Masaryk University. Her research interests include data analysis, machine learning, and their application in life sciences.

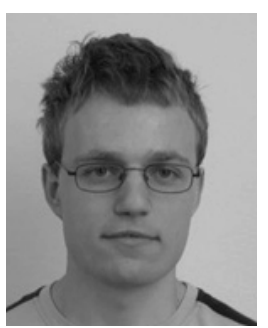

Jaroslav Šeděnka received the B.Sc. degree in computer science and the M.Sc. degree in mathematics from Masaryk University, Brno, Czech Republic. $\mathrm{He}$ is currently pursuing the Ph.D. degree with the Department of Mathematics and Statistics, Masaryk University. His research interests include lattice cryptography, applied cryptography, and algebraic number theory.

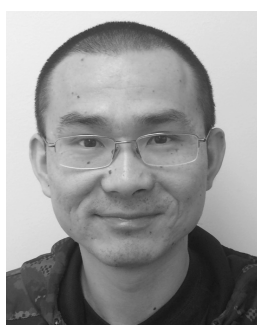

Qing Yang received the B.S. degree from the Civil Aviation University of China, in 2003, and the M.S. degree from the Chinese Academy of Sciences, in 2007. He is currently pursuing the Ph.D. degree with the Department of Computer Science, College of William and Mary. His research interests are smartphone security and energy efficiency.

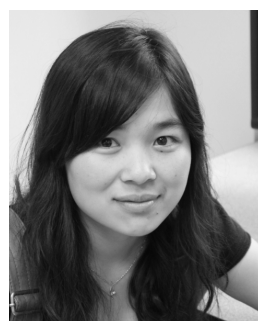

Ge Peng received the B.S. degree from the National University of Defense Technology, in 2008. She is currently pursuing the Ph.D. degree with the Department of Computer Science, College of William and Mary. Her research interests include wireless networking, smartphone energy efficiency, and ubiquitous computing.

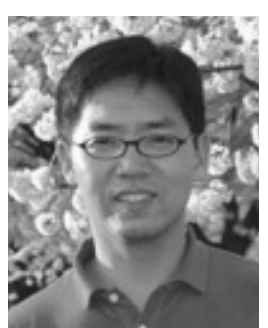

Gang Zhou (SM'13) received the Ph.D. degree from the University of Virginia, in 2007. He is currently an Associate Professor with the Computer Science Department, College of William and Mary. He has published over 70 conference and journal papers in the areas of sensors and ubiquitous computing, mobile computing, body sensor networks, and wireless networks. The total citations of his papers are more than 4800 according to Google Scholar, among which five of them have been transferred into patents and the MobiSys'04 paper has been cited more than 800 times. He is currently serving in the Journal Editorial Board of the IEEE INTERNET OF THINGS and Computer Networks (Elsevier). He received an award for his outstanding service to the IEEE Instrumentation and Measurement Society in 2008. He also won the best paper award of the IEEE ICNP 2010. He received an NSF CAREER Award in 2013. He received a 2015 Plumeri Award for Faculty Excellence. He is a Senior Member of the Association for Computing Machinery.

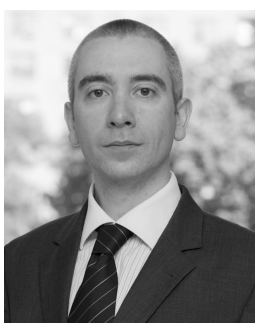

Paolo Gasti (M'14) received the B.S., M.S., and $\mathrm{Ph} . \mathrm{D}$. degrees from the University of Genoa, Italy. He was a Research Scholar with the University of California, Irvine. He is currently an Assistant Professor of Computer Science with the School of Engineering and Computing Sciences, New York Institute of Technology. His research focuses on behavioral biometrics, privacy-preserving biometric authentication and identification, secure multiparty protocols, and network security. His research has been sponsored by the Defense Advanced Research Project Agency and the U.S. Air Force. He is a Fulbright Scholar.

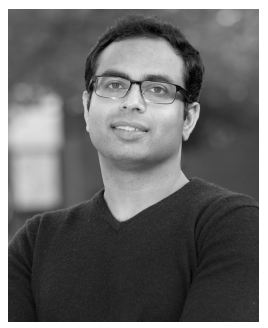

Kiran S. Balagani (M'09) received the B.S. degree from Bangalore University, India, and the M.S. degrees and the Ph.D. degree from Louisiana Tech University. He is currently an Assistant Professor of Computer Science with the New York Institute of Technology. His research interests are in cyberbehavioral anomaly detection (e.g., unauthorized user-access behaviors), behavioral biometrics, and privacy-preserving biometrics. His work has appeared in several peer-reviewed journals, including the IEEE TRANSACTIONS ON PATTERN ANALysis AND MaChine InTELLIGENCE, the IEEE TRANSACTIONS ON INFORMATION FORENSICS AND SECURITY, the IEEE TRANSACTIONS

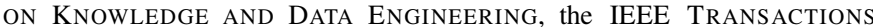
on Systems, Man, And Cybernetics, and Pattern Recognition Letters. He holds three U.S. patents in network-centric attack detection. His teaching interests include development of graduate and undergraduate courses in network security and biometrics. 